\title{
Music Recommendation Algorithms: Discovering Weekly or Discovering Weakly?
}



Jennie Silber

Muhlenberg College, Allentown, PA, USA

Media \& Communication Department

Undergraduate High Honors Thesis

April 1, 2019 


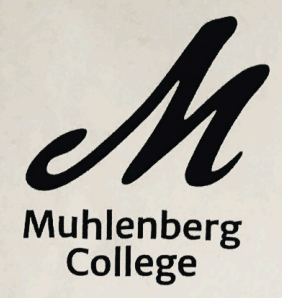

TO: $\quad$ Jefferson Pooley, Chair, Department of Media and Communication Karen Schall, Associate Registrar

FROM: $\quad$ Irene Chien, Director, Honors Program, Department of Media and Communication

DATE: $\quad$ April 12, 2019

RE: Departmental Honors in Media and Communication

CC: $\quad$ Bruce Anderson, Dean of the College for Academic Life

Amanda Jenkins-Ford, Administrative Assistant, Media and Communication Jennie Silber, Class of 2019

This letter certifies that Jennie Silber successfully defended her Media and Communication thesis, "Music Recommendation Algorithms: Discovering Weekly or Discovering Weakly?" on April 12, 2019.

In addition, Jennie Silber's Media and Communication major G.P.A. exceeds 3.7; her overall G.P.A. exceeds 3.6.

Therefore, the undersigned faculty hereby agree to award H/GH HONORS in Media and Communication to Jennie Silber.
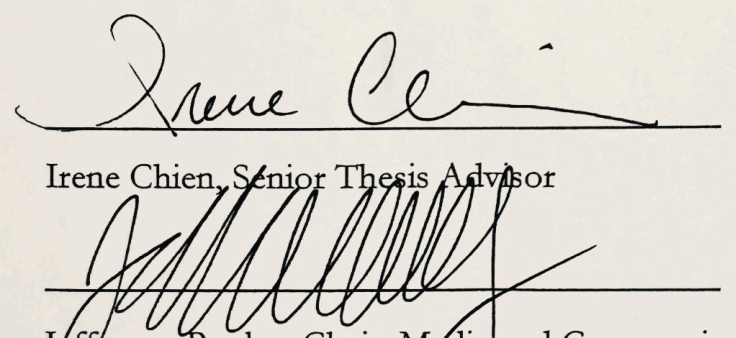

Jefferson Pooley, Chair, Mqdia and Communication \& Thesis Committee Member then $\alpha$ fuee.

John Sullivan, Thesis Committee Member 


\section{MUSIC RECOMMENDATION ALGORITHMS}

\section{Abstract}

This thesis analyzes and assesses the cultural impact and economic viability that the top music streaming platforms have on the consumption and discovery of music, with a specific focus on recommendation algorithms. Through the support of scholarly and journalistic research as well as my own user experience, I evaluate the known constructs that fuel algorithmic recommendations, but also make educated inferences about the variables concealed from public knowledge. One of the most significant variables delineated throughout this thesis is the power held by human curators and the way they interact with algorithms to frame and legitimize content. Additionally, I execute my own experiment by creating new user profiles on the two streaming platforms popularly used for the purpose of discovery, Spotify and SoundCloud, and record each step of the music discovery process experienced by a new user. After listening to an equal representation of all genre categories within each platform, I then compare the genre, release year, artist status, and content promotion gathered from my listening history to the algorithmically-generated songs listed in my 'Discover Weekly' and 'SoundCloud Weekly' personalized playlists. The results from this experiment demonstrate that the recommendation algorithms that power these discovery playlists intrinsically facilitate the perpetuation of a stardriven, “winner-take-all” marketplace, where new, popular, trendy, music is favored, despite how diverse of a selection the music being listened to is.

The content of this thesis is significant to understanding the culture of music streaming and is also contributory to the field of media communication. Unlike any other scholarly research, the "walk-through" experiment uniquely tracks a new user experience through the cognizant application of user actions and directly assesses the factors that challenge successful music recommendation. This method of research specifically highlights the influence that music 


\section{MUSIC RECOMMENDATION ALGORITHMS}

streaming platforms have not only as tastemakers, but more importantly, as gatekeepers of cultural information, shaping the perceived value and relevance of artists and genres through recommendation. This thesis underlines the challenges faced by recommendation systems in providing the novel, yet relevant recommendations necessary to satisfy the needs of users, while also providing wide-ranging, yet representative recommendations to stimulate diversity and creativity within society.

These challenges include the subjective organization of songs and genres within a platform's interface, the misrepresentation of songs and artists within genre-based playlists, the use of user actions (skips, likes, dislikes, passive listening, drifting, etc.) as an assertion of one's likes and dislikes, as well as the manipulation of hit-producing market trends. This thesis delves deeply into each challenge and the ways they affect the inaccuracy, subjectivity, and homogeneity currently projected through music streaming recommendations. Lastly, this thesis addresses the potential benefits and apprehensions of future contextually aware technology and its ability to reshape the way recommendation algorithms gather and process user listening data. Ultimately, my hope is that this research sheds light on the responsibility of music listeners, but more importantly, of music distributors and curators, as taste-makers and gatekeepers, to act progressively and ethically in constructing the cultural reality we live in. 


\section{MUSIC RECOMMENDATION ALGORITHMS}

\section{Introduction}

Music is not just a universal language that is woven into the fabric of everyday life, but it is also a deeply personal experience. As both an individual and social experience, music is among the top things that humans consume. Consuming music is not solely about the songs we enjoy listening to, but it's more profoundly about an expression of identity and connecting sound with memories and feelings. As a part of our shared and private spheres, music selection is largely dependent on where we are, who we are with, what we are doing, and how we are feeling, making it extremely important to contextualize music within everyday life. Music streaming platforms have come to realize the importance of contextualization in music recommendation and have strived to create a digital space that satisfies the tastes and preferences of each user.

Music recommendation also provides users with a condensed and tailored selection of music, saving users the time and energy necessary to discover songs and artists on their own. "Charts are among the most successful discovery features. It's not only due to a lack of time to explore the long tail, nor the fear of missing out, that is driving people to listen to hits; it's also because social proof and familiarity are quite reassuring when it comes to uncover something new" (Knibbe, 2017). While humans crave to discover new and exciting music, they are also intrinsically drawn to the familiar, unless shown otherwise. Music streaming platforms have not only hired curators to organize lists of songs for genre- and mood-based playlists, but have also relied on problem-solving operations, known as algorithms, to assemble playlists dedicated to the music discovery needs of each user. According to an industry estimate, "1 out of every 5 plays across all streaming services today happens inside of a playlist” (Ugwu, 2016), whether 


\section{MUSIC RECOMMENDATION ALGORITHMS}

platform-curated or user-curated. This format of music listening has not only shifted the way artists produce and distribute music but has transformed the way music is consumed.

In this so-called "streaming era", music streaming has emerged as a major force in the domination of digital audio consumption and continues to grow exponentially. According to global streaming statistics, there were 180.3 million music streaming subscribers worldwide in 2018, which is projected to reach 221.5 million by 2020 (Global streaming, 2017). The majority of music streaming listeners consume music through platforms like Spotify, Apple Music, SoundCloud, Pandora, Google/YouTube Red, Amazon Music, Tidal, Deezer, and iHeartRadio. As of 2018, SoundCloud amassed at least 175 million registered users with Spotify close behind with about 170 million users (McIntyre, 2018). While these two services have acquired at least 70 million users over the other music streaming platforms mentioned, the statistics are much different for paid subscribers. "An estimated 51 million people in the U.S. are now paying monthly subscriptions for music streaming services - nearly double the number of subscribers at the end of 2016," and collectively generate " 65 percent of recorded-music revenue in the U.S. in 2017” (Hu, 2018). Spotify and Apple Music have reportedly accumulated over 20 million paying subscribers, with Pandora behind at 6 million (Hu, 2018). SoundCloud has yet to amass anywhere close to the amount of paid subscribers as the rest of these platforms have, being that they only recently transitioned from a free model to the freemium/premium model popularized by most other major platforms. The major streaming services have realized that it is vital to their survival to convert free users into paying consumers. This strategy is especially difficult being that "the free-to-paid streaming user conversion ratio in the U.S. is still north of 3:1" (Hu, 2018). Many music listeners, especially among older generations, still listen to music through physical formats, like CDs and vinyl, AM/FM radio, and other free services, like YouTube or freemium 


\section{MUSIC RECOMMENDATION ALGORITHMS}

versions of Pandora, Spotify, or SoundCloud. However, now more than ever, music streaming platforms have been able to provide an immense amount of high quality content for users and have found a way to use technological and promotional incentives as a means of distribution. Many platforms have converged technologically, by implementing their services into smart devices, including cars and televisions, and have also partnered with other services, including television streaming subscriptions, like Hulu and Showtime. Music streaming has also transformed into a social practice, where users are able to connect their accounts with their social media profiles and interact with one another. This network of platforms has not only created a larger sense of community, but has also furthered users' ability to share and discover music.

For years, many companies have competed in this cut-throat industry and made substantial efforts to differentiate themselves from one another through various marketing strategies and innovative features. Specifically, the enhancement of recommendation features has been a priority for music streaming platforms. Unlike the recommendation models of other consumed media, including movies and books, music recommendation is distinguishable in its duration, magnitude, consumption behavior, and context. While movies and books take considerable time to consume and have much smaller catalog sizes, music items are usually consumed within a few minutes and are therefore, more plentiful in catalog size (Schedl et al. 2018). Additionally, music is more frequently consumed sequentially, rather than solitarily, for example, in a listening session or playlist. Due to these indispensable characteristics, music is more commonly consumed passively as well as repetitively. Most importantly, however, is that music serves a multitude purposes and has exorbitant power over mood and emotion regulation, as well as personal identity.

Considering the overwhelming amount of people that rely on streaming services for music consumption, popular platforms like Spotify, SoundCloud, Apple Music, and Pandora should be 


\section{MUSIC RECOMMENDATION ALGORITHMS}

held accountable for providing a listening experience that is attuned not only to a global market, but also to the texture of daily human experiences. While certain platforms believe that optimizing user experience entails purely algorithmic and deep machine learning systems, others are confident that human curation is the most optimal, and many provide a combination of both. Both algorithmic and human curation are critical for directing audience flows to specific artists and music genres, which can have profound effects on the cultural diversity and representation of music and artists in an online era. The recommendations made by these systems "have the power to impose and normalize certain modes of contextualization" over others (Ashers, GrünewaldSchukalla, Lücke \& Rauch, 2018). While the implementation of the "personalized music discovery playlist" has further diversified the music consumed and adapted into the taste profiles of individual listeners, there are still intrinsic challenges within the business models of streaming platforms that threaten the reliability and consistency of recommendation systems. This thesis will aim to not only analyze and assess the ways in which the top music streaming platforms construct and implement recommendation algorithms, but also evaluate how these algorithms socially, economically, and culturally shape the modern culture of music listening, and particularly, the ways that users discover music and artists.

\section{Music Industry History}

In order to understand the impact that music streaming platforms have had on music listening culture, it is vital to first understand the history that has laid the foundation of its existence. For a little over a decade, music streaming platforms have been considered a primary source of not just music listening, but music consumption, providing users with free and premium accessibility to vast libraries comprising over 30 million songs. As the internet has continued to advance and extend its accessibility, countries all over the world have begun to 


\section{MUSIC RECOMMENDATION ALGORITHMS}

embrace the use of platforms, creating a space for people of all demographics and experiences to participate in content creation and consumption. Streaming services have not only made an abundant amount of music more accessible than ever before, but have also emerged into critical outlets for music discovery and artist promotion.

The idea of music listeners executing a financial transaction in return for online music is fairly new to the industry. For close to two decades prior to the launch of streaming platforms, the music industry endured huge monetary deficits due to mass sharing of illegal music among peers (McIntyre, 2018). As a result of peer-to-peer file sharing, artists, songwriters, publishers, and labels lost a majority of distribution control and the industry suffered major losses. In 1999, the two largest independent piracy sites, Napster and LimeWire, surfaced, providing an immense library of counterfeit and bootleg tracks. It became as easy as one mouse click to download music illegally through any computer, damaging the economic prosperity of record stores, artists, and a majority of music industry employees. In 2000, several major recording companies in the Recording Industry Association of America (About RIAA) sued Napster on the grounds of copyright violation and infringement under the United States Digital Millennium Copyright Act. As a major facilitator to record labels and artists, the RIAA works "to protect the intellectual property and First Amendment rights of artists and music labels; conduct consumer, industry and technical research; and monitor and review state and federal laws, regulations and policies" (About RIAA). They not only sued pirate companies like LimeWire and Napster, but also went after individual users who facilitated this file-sharing culture. One source claims that "up to 30,000 people in America were sued at one time for illegally downloading music, with the RIAA specifically targeting colleges and universities, as there were many young people who loved music and who were acquiring their favorites for free" (McIntyre, 2018). Though this 


\section{MUSIC RECOMMENDATION ALGORITHMS}

instant and rapid means of music sharing facilitated a culture of perpetual music discovery, especially among younger generations, music is not a commodity that should be stolen or available for free use, but should be paid for like any other form of entertainment and invested in by fans. Ultimately, the RIAA was able to shut Napster down in 2001, which helped alleviate the bleeding of cash within the industry.

Despite the severity of these legal actions, illegal file-sharing culture did not cease all together. However, the music industry quickly realized that they could not afford to continue going after each and every illegal downloader and file-sharer. There needed to be a better outlet for fans to access and discover new music. In April of 2003, Apple Inc. believed they could turn the fate of the music industry around with the first marketable mp3 download technology, known as the iTunes store, and the development of a digital multimedia player with capabilities of storing thousands of songs, known as the iPod. This platform allowed for users to download digital copies from its immense virtual library as well as burn CDs into organized playlists. As the first of its kind in terms of music importation, specialized playlists, and library sharing, iTunes quickly became the most popular media library in the world.

Although this Apple-generated platform managed to minimize a decent amount of peerto-peer file sharing culture, the introduction of music streaming services in the early 2000s was ultimately the saving grace to the industry's survival. Streaming platforms like Pandora in 2005, SoundCloud in 2007, Spotify in 2008, and Tidal and Apple Music in 2015, emerged and have dominated the industry ever since. Similar to iTunes, these platforms have allowed listeners to access a vast library of music, but rather than requiring listeners to pay for and download individual files one by one, they provide a subscription-based service for users to access as much music as they'd like, whenever and wherever they'd like. These services have not only digitized 


\section{MUSIC RECOMMENDATION ALGORITHMS}

the traditional physical distribution and release of music, but have completely revolutionized music consumption from a culture of purchasing and owning to a culture of accessing and consuming (Schedl et al. 2018). While many services provide the option for users to access their music for free, platforms aim to incentivize users to pay a subscription-based fee for premium versions of their services. These incentives usually include the ability to avoid ads between songs, create and shuffle playlists, play any song on demand, listen to music offline, access promotional features, and much more.

Not only are streaming services engineered with a seemingly infinite amount of content and user-friendly functionality, but they are inherently social, allowing fans to actively share their likes and dislikes and artists to access the listening behavior data of their fans. Streaming platforms not only provide insight into audience composition and listening pattern statistics, but also ways to promote music through playlists and various forms of advertisement. Streaming platforms also give artists and songwriters a portion of revenue. While creators consider this a big step forward from the era of Napster and LimeWire, an artist's earned revenue per play is trivial compared to the return artists would normally see from physical sales back in the 1990s. The highest pay per play in the industry thus far is provided by Tidal, created and owned by JayZ, paying artists nearly a penny for every stream (McIntyre, 2017). The bulk of streaming services offer only fractions of a cent per play, with Apple Music, Amazon Music, Google Play, and Deezer (a French-based streaming service) compensating artists between $\$ 0.006$ and $\$ 0.008$ per stream, Spotify at $\$ 0.00397$ per stream, Pandora at $\$ 0.00134$ per stream, and Youtube at a mere $\$ 0.00074$ for each stream (Owsinski, 2018). SoundCloud, pays around $\$ 0.007$ per stream, however, only a small fraction of artists qualify for compensation (Ingham, 2016). Though SoundCloud has expressed the intention to expand their pool of paid artists, their current policies 


\section{MUSIC RECOMMENDATION ALGORITHMS}

stipulate that paid artists are required to be over the age of 18 , pay a fee of $\$ 8-\$ 16$ per month, get over 5000 streams per month, and only publish original music with no copyright strikes against them (SoundCloud Premier, n.d.). A major difference between SoundCloud and other major platforms is that SoundCloud artists who qualify are paid every time a user clicks the play button on their track, while most services consider a stream to be every time a user plays a track for over 30 seconds (Stats basics, n.d.). Therefore, there is a much higher opportunity for compensation for paid SoundCloud artists. While pay-per-play is an extremely important factor in an artist's career, artist exposure on streaming platforms is a major catalyst to acquiring fans and building a sustainable career. This means that platforms now have the primary power to not only distribute music to people, but also to influence what music is prioritized, primarily through recommendations.

\section{The Evolution of Taste-making}

The music we listen to is intimately attached to our identity, memories, aspirations, and daily lives. "One's taste in music has thus long been seen as a window into one's sense of self, and place in society" (Prey, 2018). As a society, we have continuously looked to others to both validate specific "tastes" and inspire us with new "tastes." Predominantly, people have looked to "tastemakers" to provide trusted music recommendations. Over time, tastemakers have taken the shape of many different forms. The first tastemakers emerged in the 1930 s with the rise of radio broadcasters and programmers, who later became known as 'disc jockeys.' "In the days before station-controlled playlists, a DJ's music selection was often guided by their personal tastes. This culture of music recommendation played a big role in exposing rock and roll artists to large, national audiences," (The history of DJing, 2012) including the introduction of the notable, rock and roll artist Elvis Presley. Beginning in the 1950s, DJs began appearing outside of radio 


\section{MUSIC RECOMMENDATION ALGORITHMS}

stations, performing at live venues or parties. "The appreciation of cultural intermediaries was often linked with an idea that a good recommendation is not only based on personal preferences but on the idea of curation, that is, certifying and validating the content for the audience" (Kuoppa, 2018, p. 52). Alternative cultural intermediaries emerged in the late 1960s, with the popular music magazine, Rolling Stone, which inspired other magazines like Spin and Clash. These magazines typically included news related to music, interviews, record reviews, concert reviews, and occasionally a cover mount with recorded music (Wikipedia contributors, 2018). Also during this time, a massively popular television program, known as American Bandstand, emerged, creating an exciting and entertaining variant of music curation, outside of its customary paper or radio format. This show incorporated a feature called "Rate-a-Record," allowing ordinary people to evaluate a record on a scale of 35 to 98 (J. McClain \& A. McClain, 2012). As television grew in scope and popularity, the evolution of the "video jockey" developed as a part of the creation of MTV (Music Television), which broadcasted popular music videos, mainly targeted towards a younger audience. Still, today, many people rely on radio and television for music discovery. One powerful tool that people have paired with radio and television music listening is the use of a mobile device app known as Shazam. Shazam allows users to identify a song by processing a short sample from a song and retrieving the song title and artist's name for the user. This technology has reformed the way people listen to radio especially, transforming users from passive to active listeners.

Another major change that mediums like radio have undergone is the transfer of control from individual DJs to larger stations and corporations. This shift in power began in the 60s and 70s, when record promoters realized that radio airplay was vital for their clients to reach the public and increase record sales. During this era, "mid-level DJs could expect to clear at least 


\section{MUSIC RECOMMENDATION ALGORITHMS}

$\$ 50$ per week in bribes, with higher-profile jocks commanding much higher prices and much flashier swag" (Kelly, 2016). This "soulless music landscape, wherein the tastemakers are just regurgitating whatever they've guzzled down from the hands that feed," has become popularly known as "payola" (Kelly, 2016). Payola has created a culture that has stripped radio DJs and video jockeys of their own personal expression through song recommendation, and in turn, transferred the control of music distribution from the hands of these individuals into the hands of major corporations. Although this practice is still prevalent in the radio industry today, many question if payola has transformed digitally into the music streaming industry. In order to evaluate this issue, it is important to not only look at who the tastemakers are within the music streaming industry, but also what they are capable of.

Many people still appreciate the curation of cultural intermediaries, such as radio hosts, celebrities, festival coordinators, and music bloggers, and also value the recommendations from trusted friends and family. Streaming platforms have found a way to capitalize on both the consistency of cultural intermediaries and the personalization of friends and family. With the integration of advanced algorithms and massive accumulation of artists and users, major streaming platforms have completely transformed the practice of music discovery, providing a new list of personalized recommendations for each user every single week. Not only does this feature eliminate a user's reliance on others as well as the time-consuming endeavor of searching online, but streaming services organize these recommendations into confined playlists, based on specific categorizations. "Given that the amount of music circulating on the internet increases all the time, the need for this kind of compression of cultural data and ability to find the essence becomes more focal than ever" (Kuoppa, 2018, p. 21). The consolidation of music into meaningful categories has not only made this process easier and more efficient for users than 


\section{MUSIC RECOMMENDATION ALGORITHMS}

ever before, but has created a demand and expectation for streaming platforms to serve as reliable, yet sophisticated taste-makers.

With the power to categorize and sequence music, streaming platforms take on the influential role of taste-making, but also the authoritative role of media "gatekeeping." Gatekeepers have the ability to "frame, legitimize, and value goods in order to make an impact." By estimating these values based on user data and behavior, gatekeepers may produce recommendations that highlight certain content while concealing other content. "If a piece of music is put on a pedestal, it means that this piece of art is valued somehow more than the rest and that it is taken as a representative of its culture" (Kuoppa, 2018, p. 21). The anonymous magnification and hindrance of music does not only shape the make-up of the music industry, but also influences peoples' impressions and opinions towards what kind of music is considered valuable and desirable or not. Platform gatekeepers can take form as an algorithm, human "expert," or even a mix of the two. While these recommendation systems are imperative for large-scale song recommendation, they also have profound effects on the social and cultural value of certain music, artists, and genres.

\section{Recommendation Algorithms}

Across all digital arenas, algorithms have been used to generate lists of recommended items that reflect a customer's behaviors and interests. These algorithms are commonly referred to as "black box" systems, "where users are unable to see inside the system and evaluate its core principles" (Kuoppa, p. 89, 2018). Companies treat their algorithms as commercial products, which is why they are safely guarded from rival companies and not made accessible to the public. "At Amazon.com, recommendation algorithms are used to personalize the online store for each customer, for example showing programming titles to a software engineer and baby toys 


\section{MUSIC RECOMMENDATION ALGORITHMS}

to a new mother" (Lops, Gemmis \& Semeraro, 2010, 74). Similarly, Netflix suggests new movies and TV shows by taking users' star-based ratings and other similar users' ratings to better inform their recommendations (Ciocca, 2017). Algorithms are extremely valuable to the essence of these user-centric companies. If these algorithms are able to satisfy a user's needs in terms of recommendation, users are likely to develop a trusted relationship with that company and continue to consume their products as opposed to their competitors' products.

Building relationships with music consumers is just as important for streaming platforms. While the abundant libraries of music available on streaming platforms may provide for simplified searchability and access, it can be frustrating at times to find the perfect collection of songs to listen to at the right time and in the right place. With the creation of the "playlist" feature, users are provided direct access to lists of songs that are deliberately grouped together based on some sort of association, whether that be genre, mood, style, cultural distinction, or geographic location. Many streaming services not only curate pre-set playlists for listeners but also allow users to create their own playlists and collaborate with one another across playlists. The personalization of playlists has spawned a new level of intimacy between distributor and consumer. Although a fairly recent addition to music streaming platforms, personalization has been incorporated into many other mediums, including social media, news, advertising, and online shopping, which organize information based on past user behavior. In terms of music personalization, many users feel that these platforms truly care about their preferences and know what they need to hear. However, many users aren't always aware of the effects that their listening interactions have on the makeup of their taste profile, yet expect the algorithm to contextualize these actions. The frequent miscommunication between algorithm and user makes 


\section{MUSIC RECOMMENDATION ALGORITHMS}

it extremely difficult for recommendations to continually satisfy a user's needs and maintain this trusted relationship.

Rishi Malhotra, CEO of Indian music streaming service, Saavn, articulates that “it's about the second song. The first song, you already told me... So if I get that wrong I'm pretty much dead in the water. But it's really the second song that has to have that sort of vibe to it, and that serendipity that makes you go, 'OK, cool, this thing gets it,' and people will begin to invest more to get it better...You get algorithm right and you'll start getting session length that compounds" (Croll, 2015). While Malhotra stresses the importance of the "second song," many may wonder, what makes a second, or even third or fourth, song have "that sort of vibe" or "serendipity" to it? The answer is both simple and complex. When music listeners are looking to "discover", they ideally want to be recommended songs that are "both novel and relevant simultaneously" (Celma \& Lamere, 2011, p. 58). Novelty is defined as "the opposite of the user's familiarity with the recommended items" (Celma, 2010, p. 15) and relevance is defined as "having significant and demonstrable bearing" on the recommended items (Relevant, n.d.). Conversely, users will consider recommendations that are both familiar and irrelevant to be too "popular" or "mainstream", those that are novel but irrelevant to be "random", and highly relevant but too familiar tracks to be too "safe," providing music that users like but lack the excitement that users crave. Therefore, it is difficult to achieve recommendation that perfectly balances the novel and the relevant, but it is necessary in order to secure the most platform loyalty.

\section{Platform Audiences}

It is extremely difficult to provide a gratifying and suitable service for all streamers, especially considering that not all streamers of music are "equal" in nature. In this sense, equal 


\section{MUSIC RECOMMENDATION ALGORITHMS}

means that while some users are passionate about music and love to discover new artists, others only occasionally stream music and tend to listen to the same songs over and over again. The kind of listener a person is can largely affect a user's behavior in terms of listening position, skipping ratio, average streaming session length, and variance of artists and songs listened to. These factors not only create distinct perceived values of music for every user, but also various user consumption habits across different streaming platforms.

Though most streaming platforms aim to appeal to the masses, it is apparent that most streaming listeners either stay loyal to one preferred platform or use a variety platforms for different listening purposes. As far as discovery features are concerned, public opinion tends to concur that Apple Music is the best streaming service for those that are dedicated to the 'Apple brand,' "lovers of iTunes, hand-curated live radio, and all things Apple" (Hall, 2018). Pandora is considered the best for passive listeners, "those who like to press play and walk away" due to its ability to provide radio-style streaming for endless lists of complementary songs. SoundCloud, on the other hand, has been praised for its "hands-on approach to music discovery," and its ability to empower amateur artists. The platform Tidal is perceived as the weakest among discovery features due to its focus on exclusive artists and high fidelity. Finally, Spotify is perceived as the "best music streaming service overall" in terms of discovery, due to its extremely user-friendly interface, diverse array of well-curated playlists, and "well-rounded, intuitive, and hassle-free" features (Hall, 2019). The affordances of each platform not only shape the ways in which each platform is perceived, but also construct an imagined audience for each platform. Because most streaming platforms allow for users to connect their profiles to their social media accounts, streaming has become ingrained into our online realities. Many users enjoy sharing content with their friends, collaborating on playlists, and also seeing what their 


\section{MUSIC RECOMMENDATION ALGORITHMS}

friends are listening to in real-time. While it is likely that people primarily consider their personal preferences when choosing loyalty to a streaming platform, many treat streaming services as a social network and will follow whatever is trending among their friends.

Out of the top streaming platforms listed above, Spotify and SoundCloud are the only two to offer personalized playlists solely focused on the discovery of music, which Spotify calls 'Discover Weekly' and SoundCloud calls 'SoundCloud Weekly.' Other platforms offer personalized playlists; however, they are usually tailored to specific categories, like new releases, genres and moods, or special mixes. For example, Pandora offers 60 algorithmicallycurated playlists, personalizing activity and mood-based soundtracks, including "Your Party Soundtrack," "Your Hip-Hop Soundtrack," "Your Focus Soundtrack," and many more (Dredge, 2018). Google Play, Spotify, and many others offer "New Release" playlists, which personalizes a collection of brand new tracks and is updated weekly. Apple Music calls their version of this playlist "New Music Mix" and also offers "Friends Mix," which is filled with tracks based on what your friends listen to (Hussein, 2018). Although both Spotify and SoundCloud feature discovery playlists that are virtually the same in format, interface, and algorithm, Spotify has pioneered the implementation of this personalized feature.

\section{Spotify's Discover Weekly}

In July of 2015, Spotify launched 'Discover Weekly', which they describe as “a playlist of songs we think you'll love. Based on what you and those with similar music tastes listen to, it gets even better the more you use Spotify." They also advise that "“Discover Weekly” automatically refreshes every Monday, so make sure you save the tracks you like from it before it updates" (Spotify.com, n.d.). Many avid users of Spotify, including myself, will agree that this personalized playlist definitely includes some hits that are pleasantly surprising 


\section{MUSIC RECOMMENDATION ALGORITHMS}

recommendations, but also quite a few misses, that seem to be large extrapolations from a user's listening history. In order to uncover the mysterious "magic" that powers the curation of 'Discover Weekly', I decided to look deeper into the algorithm working behind the scenes.

The development of this recommendation algorithm can actually be attributed to Spotify's acquisition of a company called Echo Nest in 2014. Echo Nest provides Spotify with an intense data analytics infrastructure necessary to analyze the audio and textual content of each piece of music. Using this technology, Spotify's 'Discover Weekly' algorithm considers not only an individual's personal music selection, but at it's core, the selections of other, similar people. "Spotify begins by looking at the 2 billion or so playlists created by its users - each one a reflection of some music fan's tastes and sensibilities." (Pasick, 2015). Spotify will take songs you have listened to and saved and then combine your own taste profile with similarly categorized songs that appear on playlists of others with alike taste profiles. This method is known as collaborative filtering.

Before the implementation of collaborative filtering became popular, many platforms relied on the use of content filtering, or the process of recommending items based on the similarities and differences between items a user has liked in the past. For example, Pandora is powered by a content-based recommender database called the 'Music Genome Project,' which organizes music by its traits, or "genetic makeup" (Prey, 2018). The taxonomy of these genetics can include broad musical concepts like song structure, rhythm, and instrumentation to much more detailed information, such as gender of the lead vocalist, tempo of the chorus, and distortion of the electric guitar. Depending on the complexity and subsets within a specific genre, the number of genes assigned can vary. "Rock and pop songs have 150 genes, rap songs have 350, jazz songs have approximately 400, while world and classical music have between 300 and 


\section{MUSIC RECOMMENDATION ALGORITHMS}

500 genes” (Prey, 2018). Through an extremely labor intensive process, Pandora employees manually assign each attribute or "gene" a number between 0 and 5 (in half-integer increments) based on specific similarities, and an algorithm is then used to compare the ratings of each song to the genetic makeup of every other song in its database. The songs that match the closest in rating makeup a new Pandora “station.” Based on a user's feedback (thumbs up or down), the algorithm will instantly adjust its recommendations by "raising the weights of genes that are important to the individual and reducing the weights of those that are not" (Prey, 2018). While Pandora's content-based filtering can process an elaborate web of musical elements, this method is manually time-consuming, reliant on user action, and disregards the cultural and social landscape of music listening.

Rather than filtering through explicit feedback, collaborative recommendation systems are based on implicit feedback, or the assumption that people like things similar to other things they like and therefore, like things that are liked by other people with similar taste. "Spotify considers everything from professionally curated playlists like 'RapCaviar' to your cousin Joe's summer barbeque jams. It gives extra weight to the company's own playlists and those with more followers. Then it attempts to fill in the blanks between your listening habits and those with similar tastes" (Pasick, 2015). The goal of this algorithm is to find songs that you will like, but have not yet listened to, or as previously mentioned, recommend a list of 30 songs that are both "novel" and "relevant" (Celma \& Lamere, 2011, p. 58). The image below is an illustrative depiction of what Spotify's collaborative filtering looks like in its most basic form. 


\section{MUSIC RECOMMENDATION ALGORITHMS}

Figure 1: Collaborative Filtering



Image via Erik Bernhardsson

Monday, tanury 13, 14

Image source: Collaborative Filtering at Spotify, by Erik Bernhardsson, ex-Spotify.

While one character expresses his interest in tracks "P, Q, R, and S" and the other in tracks “Q, R, S, and T," they both recommend that the other consider listening to the track that the other one had not mentioned; track P and track T. It is assumed that because both characters enjoy tracks Q, R, and S, they have a similar taste profile and will therefore enjoy tracks that others associate with the tracks they already like. However, rather than just one taste profile influencing the recommendations of another profile, streaming platforms amass a large database of users that then generate an immense amount of listening data, which algorithms process into an infinite network of connections and associations. Spotify, in particular, houses nearly 100 million users along with a library of 30 million songs. Platforms with this large of a database often use computational clusters that aid their algorithm in storing and analyzing huge amounts 


\section{MUSIC RECOMMENDATION ALGORITHMS}

of unstructured data (Rohan, 2018). The intricate and complex analysis of pattern detection has not only been a salient asset of recommendation algorithms, but has played a critical role in changing the way consumers interact with and experience music.

Collaborative filtering, on its own, cannot provide for satisfactory recommendations. This is due to a "cold-start problem", where conditions are not yet optimal for the platform to provide the best, or most accurate results (Galvanize, 2016). This problem can be relevant for both new users, who have not yet listened to enough of what they like, or for a new artist or song, that has little to no generated user data, and can therefore, not be recommended. A lack of user data makes it virtually impossible for new and unfamiliar sounds and artists to break through into a network of potential fans. Spotify recognizes this potential flaw and with the help of Echo Nest, has also employed the use of Natural Language Processing and Raw Audio recommendation models to reverse these effects within its algorithm. Natural Language Processing "is the ability of a computer to understand human speech as it is spoken" (Ciocca, 2017). Spotify employs technology that is able to scan the web in search of artist names and song titles. These mechanisms find "which adjectives and what particular language is frequently used in reference to those artists and songs, and which other artists and songs are also being discussed alongside them" (Ciocca, 2017) in order to determine correlation and relevance. Theoretically, Natural Language Processing is comparable to the concept of a word map (as pictured in Figure 2), where information is conceptually organized and defined by relational terms. 


\section{MUSIC RECOMMENDATION ALGORITHMS}

Figure 2: Natural Language Processing

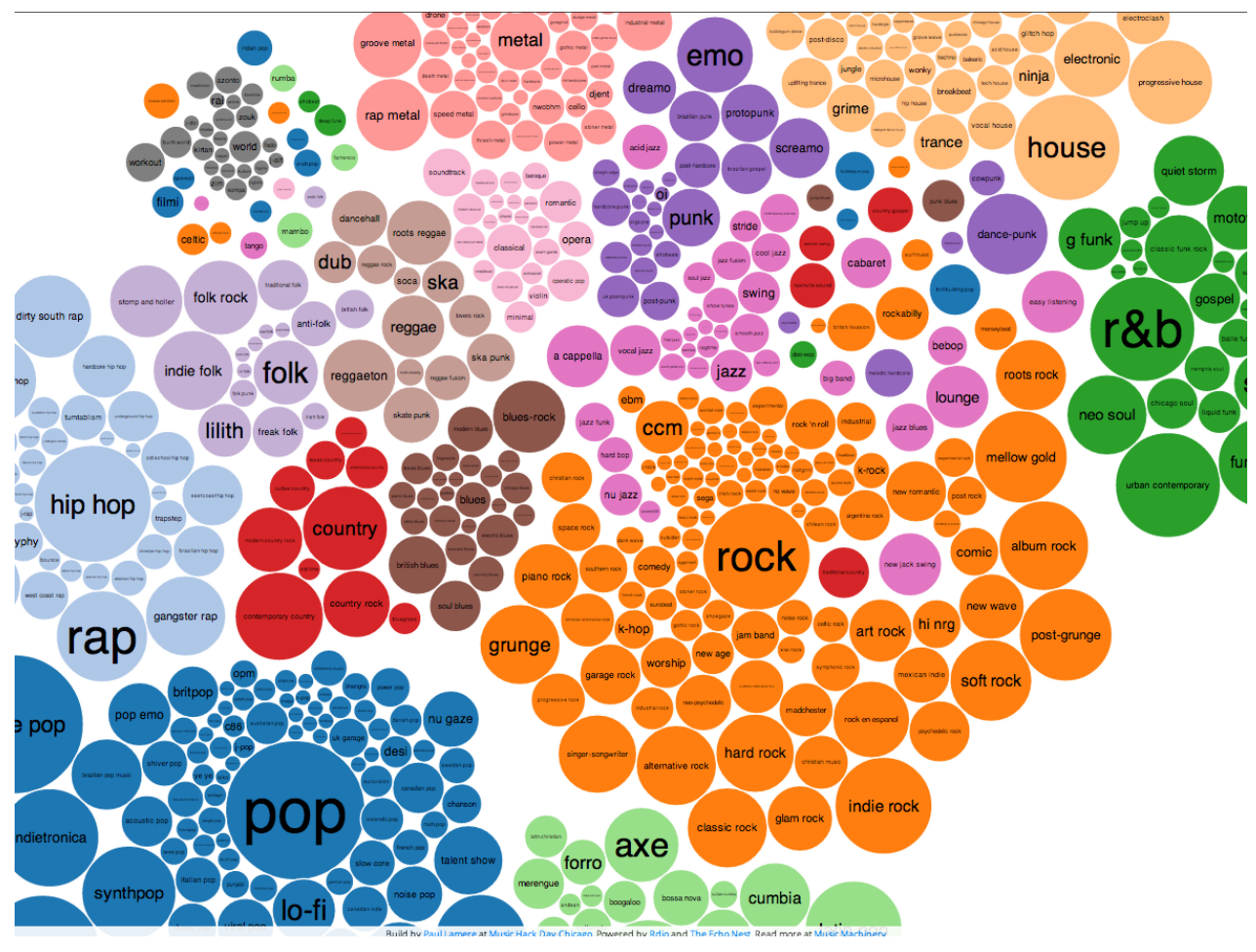

Image source: Music Popcorn - A visualization of the music genre space, by Gene Vincent

Lastly, Spotify integrates raw audio models, which particularly help account for new songs that are relatively unknown by the public. "Convolutional neural networks are the same technology used in facial recognition software. In Spotify's case, they've been modified for use on audio data instead of pixels" (Ciocca, 2017). Through convolutional neural networks, musical elements like time signature, key, mode, tempo, and dynamics can be detected and analyzed to associate songs that have similar acoustic patterns (Galvanize, 2016). Some sources even believe that these neural networks have the ability to pick up certain chord progressions in relation to specific genres (Novet, 2014). Though the processing of raw audio models isn't a perfectly refined machine nor void of error, the possibility of accurately detecting patterns as intricate as chord progressions could potentially provide meaningful knowledge to both playlist curation and 


\section{MUSIC RECOMMENDATION ALGORITHMS}

genre classification. Spotify executives express, "We now have more technology than ever before to ensure that if you're the smallest, strangest musician in the world, doing something that only 20 people in the world will dig, we can now find those 20 people and connect the dots between the artist and listeners" (Pasick 2015). Identifying certain key characteristics in music is extremely important for the purpose of recommending new or lesser-known artists. The chart below (Figure 3) conveys a visual representation of the multiple acoustic layers of four aurally diverse compositions, divided by specific acoustic elements. The orange-colored wavelengths depict the aural differences between the pieces of music. It can be assumed that songs that are more alike in neural network configuration will be categorized together by the recommendation algorithm.

Figure 3: Convolutional Neural Networks

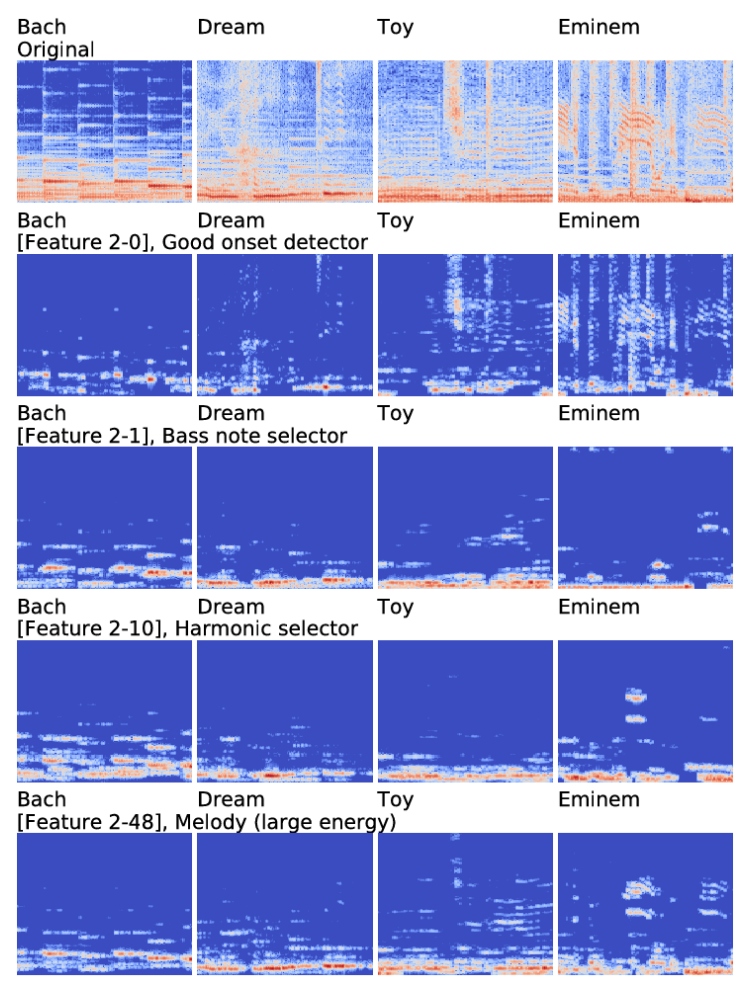

Image source: Explaining deep convolutional neural networks on music classification, by Keunwoo Choi 


\section{MUSIC RECOMMENDATION ALGORITHMS}

While the mechanisms listed above have the ability to detect similarities between songs, they are also programmed to detect and eliminate outliers. Outlier detection is actually a common practice used in financial security, in that algorithms are the tools used to detect fraudulent charges, or those that seem out of the ordinary (Galvanize, 2016). In music recommendation, if you normally listen to Hip-Hop and R\&B artists, your 'Discover Weekly' playlist won't include country songs if your friend plays a Florida Georgia Line song to try and convince you that country music is "good." However, if your friend is successful in their pursuit and you do start consistently listening to country music, the recommendation algorithm will likely begin to include country selections in your 'Discover Weekly' playlist. It is also possible that after a few weeks, if you decide you can't stand country music anymore, it will be difficult for the algorithm to discern your actual preferences for a period of time. This is why platforms like Spotify encourage their users to like and save songs to playlists that they enjoy listening to and dislike and skip songs that they don't enjoy. Despite the possibility of inaccuracy and miscalculation, Spotify's recommendation algorithm has proved to be the most successful in its pursuit of understanding the complexity of music taste and has paved the way for many other platforms to personalize their services.

\section{SoundCloud Weekly: a page from Spotify's playbook}

About four years after Spotify first launched its 'Discover Weekly' feature, music streaming platforms like SoundCloud, Apple Music, and Pandora saw its success and decided to jump on a similar band wagon. SoundCloud's version, 'SoundCloud Weekly', was released on September 10, 2018 and practically imitates Spotify's personalized recommendation model and playlist interface. SoundCloud's CEO, Kerry Trainer claims that "SoundCloud has the largest, most diverse music catalog ever assembled" (Seto, 2018), with 30 million creators and over 180 


\section{MUSIC RECOMMENDATION ALGORITHMS}

million tracks, as compared to Spotify's 35 million tracks (Korstanje, 2018). SoundCloud's strategic positioning in music discovery not only provides a much larger pool of artists for their algorithm to sort through and recommend to listeners, but also greater pool of independent artists than ever before, differentiating this platform from all others. Due to SoundCloud's user-centric business model, any user is allowed to and encouraged to be an artist and directly post music to its public platform.

SoundCloud is built upon the idea of "community" and being a part of this community means that users of all levels of recognition and musical talent are encouraged to create content and support one another through likes, genuine comments, and re-posting, generating further circulation of music. Re-posting a track has been extremely powerful for SoundCloud artists to gain attention from a variety of audiences and puts a great deal of algorithmic control into users' hands. It appears that the more active an artist is in supporting other artists, the more exposure they receive, and the better the algorithm will work for them. Other factors that can boost a SoundCloud artist are labeling and tagging their own tracks accurately and concisely, including high-quality links, and investing in aesthetically pleasing album art. It is also popular for artists to buy SoundCloud plays, just as social media influencers purchase likes, in order to increase their following. One source even suggests that "Buying social proof as you promote yourself makes it look to your potential fans like you're growing organically" (Max, 2014). While the culmination of these self-promotion strategies give artists a better chance of getting into the pool from which music is selected, they aren't necessarily the most ethical ways of being discovered.

There are, however, genuine, authentic strategies that an artist can use to amplify their content. For one, they can utilize "waveform comments to tell your fans and community about your process." SoundCloud blogger, Rory Seydel encourages artists to "be transparent about how 


\section{MUSIC RECOMMENDATION ALGORITHMS}

you made your track. Ask for feedback and mention specific sections” (Seydel, 2016).

Additionally, sharing another artist's music can create a relationship between you and that artist, increasing the chances that they will also share your content. These relationships can "lead to gigs, collaborations and helpful partnerships. All that good community stuff” (Seydel, 2016). SoundCloud creators have the ability to upload content freely without the expensive record deal or distribution plan typically necessary for most competing streaming services. "It's music interaction and discovery distilled to its purest form, home to just as many famous artists as ones that will be soon. It's as close to indispensable as you get on the internet today" (Horn, 2014).

Despite SoundCloud's sincere intentions to uphold its platform for grassroots musicians and connect these artists with potential fans, personalized discovery playlists like 'SoundCloud Weekly' have unintentionally exposed SoundCloud's ongoing struggle with copyright management (Deahl, 2018). Due to the fact that music streaming platforms have become the central source of distribution and music industry revenue, content owners are now paying much more attention to the distribution of illegal content. "Its inability to deal with sampling and remix culture led to a heavy hand with doling out strikes — SoundCloud's term for instances of copyright infringement — with a system that was (and still is) unreliable, despite efforts to allow some gray area content like DJ mixes" (Deahl, 2018). Music producer, DJ, and journalist, Dani Deahl, explains that her "current SoundCloud Weekly playlist does showcase some legitimate underdog tracks with relatively few plays, but it's also serving me [her] mashups that violate SoundCloud's own terms of use" (Deahl, 2018). In March of 2017, SoundCloud signed contracts with the three major record labels, Universal, Sony, and Warner, and clearly stated that "users must not upload to the public any content to which you do not hold the necessary rights" (Deahl, 2018). Many rights owners have pursued actions to ensure the removal of illegal mashups and 


\section{MUSIC RECOMMENDATION ALGORITHMS}

remixes on SoundCloud. This changing culture has prompted many independent creators to upload elsewhere in the streaming community. In recent years, Spotify has attempted to combat copyright infringement with an anti-piracy algorithm, meant to mitigate illegal downloads. However, this algorithm has actually been criticized for taking down music that wasn't actually illegal. While it is vital that artists' and songwriters' rights are not being violated, it seems that consequentially, SoundCloud's intended community-centric business model may not be able to nurture the artistic visions of all members of its community.

However, within the past few years, this copyright management problem has been one of the least of SoundCloud's worries. In July of 2017, "SoundCloud announced that it was laying off 173 employees, about 40 percent of its work force" (Wortham, 2017), and realized they would go bankrupt if they couldn't find a way to monetize their business. Consequently, SoundCloud reported that instead of "trying to mimic Spotify," they needed to focus on selling music creation tools to independent artists (Sanchez, 2018). While acclaimed rapper, Chance the Rapper, was able to use his status and fan base to attract more users and champion independent artists, SoundCloud still needed to find a way to fund and monetize their business. In hopes to generate capital-efficient growth, SoundCloud decided to follow the same path as the other major platforms, leading to the creation of Premier subscription tiers, Pro and Pro Unlimited, for artists, and tiers, Go and Go+, for users. "SoundCloud Premier is supposed to be the platform's solution for members to make money from their music directly, but years later, it's still invite-only" (Deahl, 2018). While SoundCloud's mission has been to provide an equal opportunity for artists of all statuses, an artist with Premier access is given many more benefits and more likely to reach potential fans. SoundCloud also recently announced that it is adding distribution to its Premier program, allowing those eligible to "self-upload, monetize, and publish their own songs to other 


\section{MUSIC RECOMMENDATION ALGORITHMS}

streaming platforms and outlets such as Spotify, Apple Music, Amazon, Facebook, Instagram, iHeart Radio, and Pandora", all from within SoundCloud (Deahl, 2019). “The cheaper SoundCloud Pro tier costs $\$ 72$ annually, and it only allows you to distribute one release to all major services. Pro Unlimited costs \$144 annually, and it allows for unlimited distribution” (Deahl, 2019). SoundCloud Pro and Pro Unlimited creators benefit from these subscriptions by gaining access to automatic eligibility for promotional rotation in featured playlists, along with promotion and marketing of their content, live showcases, and partnership opportunities with some of the world's top brands (SoundCloud Premier, n.d.). These extra features and opportunities can affect an artist's visibility and the ability to be "discovered." "Practically all artists that have made it big, cannot say that they've done it alone. Across the board, there was a point between the artist making great music and being boosted by fans ... and then getting picked up by someone" (Max, 2014). From the personal experience of working for a small record label focused on acquiring up-and-coming talent, I watched A\&R (artists and repertoire) teams frequently use SoundCloud's platform to discover and reach out to talented, unknown artists. Whether this "someone" is an A\&R representative, radio DJ, music blogger, Reddit curator, or YouTuber, the relevance and visibility of a small-name, independent artist within SoundCloud's playlists are vital to an artist's potential future success.

\section{Context of Skipping and Passive Listening}

The actions completed, or not completed, by users play a huge role in whether an artist is recommended to a user as well as the placement of a song within their discovery playlist. Director of the Developer Platform for Echo Nest, Paul Lamere, explains that "The skip button is now a big part of the overall listening experience" (Lamere, 2014). In Spotify's case, a skip is registered if the song is abandoned before 30 seconds of the song is played through. It is 


\section{MUSIC RECOMMENDATION ALGORITHMS}

important to note that skipping a song does not only occur when listening to an unfavorable song, but can actually acquire a variety of implications that both algorithmic and human curators can't seem to contextualize. One may skip a song because they simply don't feel like listening to that song at that particular moment in time, they feel that a particular song has been overplayed, or just because they are trying to find another track while sifting through a playlist. Many streaming platforms rely on the idea that "playing or skipping songs is the strongest signal at our disposal: the intuition is that users like songs they finish, and dislike songs they skip." While skips and plays are the closest algorithms can get to confident measures, this kind of analysis can often be misinformed. Lamere conducted a study in 2014 where he processed billions of plays from millions of unique listeners around the world. He reports that "Nearly one quarter of all song plays are abandoned in the first 5 seconds" and "The likelihood that a song will be skipped within the first thirty seconds rises to 35.05\%" (Lamere, 2014). Not only do most skips occur within the first thirty seconds of listening, but "new, unfamiliar music has a very high skip rate" (Snickars, 2017, p. 195). Consequently, it is made more difficult for lesser-known artists, particularly those that lack representation and promotional resources, to surface into well-known playlists or a large amount of music recommendations.

One reason that recommendation systems can't comprehend the enigmatic context of a skip is because users "have adopted conversive listening position in which they can shift the level of their listening awareness rapidly" (Flynn, 2016). Essentially, these systems are unable to identify whether users are listening consciously, subconsciously, or passively. Before the emergence of personalized playlists, people had to consciously identify, find, and then store their own music. "The most unpredictable narrative a user could use was the shuffle function" (Flynn, 2016). These curated personalized playlists compile a "narrative" for you and allow for users to 


\section{MUSIC RECOMMENDATION ALGORITHMS}

shift between various listening positions. Long, passive sessions are not necessarily obstructive to the service; however, it is important to recognize that the songs listened to hours into a session are less likely to resonate with a listener than songs listened to before or after an action is made by that listener (Kachkach, 2016). When a listener is not paying attention to their music, they also aren't skipping songs, which is likely to be mistaken as a positive signal to the algorithm. One listener exclaims, "When I'm listening to music, my phone usually rests in my pocket and I really can't be bothered to fish it out just to tell Apple if I love or hate a piece" (Krywko, 2016). While recommendation systems have the ability to recognize activity, such as pages viewed, songs saved, and songs skipped, it is much more difficult to distinguish active listening from passive listening. This contextual issue can be problematic for providing recommendations representative of a user's complete taste profile. For example, a playlist that is used for the purpose of background music while studying or napping is likely to be less interacted with than a playlist used during an intense workout or long road trip. Each of these activities is generally associated with specific genres, like classical music for a long study session or hip-hop for an intense workout. While streaming services encourage users to be active in their listening habits, by skipping songs you don't like, playing new music for extended periods of time, saving songs to playlists, and following your favorite artists or bands, active-specific genres are likely to generate more detailed user data than passive-specific genres. Because listening to music doesn't intrinsically require continued attention, user behavior tends to vary between passive and active listening, proposing a challenge to collaborative filtering, or the reliance on others' taste profiles, for personal discovery recommendations.

\section{Drifting Affordances}




\section{MUSIC RECOMMENDATION ALGORITHMS}

In a 2017 qualitative research study that collected data thorough semi-structured interviews, researchers found that "the most common occasion in which the agency of the algorithms actualized was when the users did not know what to listen. One of the interviewees described this moment as 'adventurous vibe'." This interviewee explained "If I'm in adventurous vibe I go to the Daily Mixes (in Spotify) or Discover Weekly. But because my listening to music is very emotionally related I tend to go to playlists, for example, if I know that I want some 70's music for driving in a car or for venturing I go there. - - But then if I have a pop-adventure feeling there are radios or "recommended for you" for it" (Kuoppa, 2018, p. 83). In addition to playlists, streaming platforms also recommend music through features like "Related Artists", "Related Tracks", "Recommended songs", "Fans also like", "For fans of...", and "More of what you like", which associate the artists or tracks that a user interacts with to artists or tracks that are usually similar in genre and popularity. These subtle features allow users to discover music either intentionally, through actively searching for similar recommended items or, in most cases, unintentionally, by listening to a song and letting the algorithm decide which songs play next. This ability to "drift" allows users to uncover new musical interests and tastes based on an algorithmically curated selection of related songs and artists. Spotify, in particular, suggests a list of "recommended songs" at the end of each self-curated playlist and includes the option of "adding to playlist" next to each recommended song. This algorithm seems to base their recommendations off of the songs within that self-curated playlist and provides song recommendations that mostly come from an artist already in that playlist or artists that fall into a similar category. If users listen to a playlist from start to end, the next songs that will play will be from the "recommended songs" list, leading users to listen to songs that aren't saved within their library and are a lot of times, unknown. A "user can listen to a playlist quite superficially and one 


\section{MUSIC RECOMMENDATION ALGORITHMS}

song can make an impact that leads the user to listen to that album the song is in" (Kuoppa, 2018, p. 87). Though many people rely on the serendipity of a "drifting affordance" when using discovery features, they still expect a sense of awareness and the ability to detect and eliminate outliers within the algorithm.

Features that afford for user drifting and exploration are also largely dependent on a responsive and sensible algorithm. SoundCloud, in particular, has struggled with the relevance and precision of their recommendations. For example, in 2016, Kanye West dropped a track titled "Real Friends" on SoundCloud. Shortly afterwards, an 18-year-old kid from Kansas City, Missouri saw his song skyrocket from 5,000 to 150,000 plays within 24 hours. "Thanks to the streaming site's "related tracks" algorithm, rapper Rory Fresco's "LOWKEY" was next in the play queue after Kanye's "Real Friends."'(James, 2017). Rory Fresco was just a kid who rapped for enjoyment and had very little exposure on the web. Due to this placement in SoundCloud's system, Fresco became the first rapper to rise to fame due to an algorithm and was later signed to a major label. These seemingly strange and incoherent algorithmic placements have become typical of SoundCloud's culture. While these unpredictable recommendations can encourage unknown artist discovery, many believe that this kind of "musical Russian roulette" has hindered SoundCloud's use as a discovery tool. Another streaming service that has faced similar backlash for its lack of recommendation awareness has been YouTube. YouTube provides a platform for endless discoveries of not just albums and audio content, but also television shows, podcasts, music videos, live streams, documentary films, and much more. However, when listening to algorithmically-curated playlists, the service will often diverge into an irrelevant selection of songs or continually play the same artists over and over again. 


\section{MUSIC RECOMMENDATION ALGORITHMS}

Whether the content that platforms "drift" to are relationally accurate or not, these features do allow for users to dive deeper than ever before into a rabbit hole of discovery. "Users will jump from artists or albums to another more often and in faster pace than before. Bad songs can be easily skipped and listening becomes fragmented since tracks are not listened to in their original context, the album format" (Kuoppa, 2018, p. 87). Single song streams and playlists have not only encouraged users to explore multiple artists at a time, but have actually become the dominant way of listening to music. In fact, in a 2016 Music Biz consumer report, "77\% of participants surveyed said that their primary form of listening was playlisting or single song streaming. Compared to just $22 \%$ who chose the album as their format of choice" (Seydel, 2017). While the standard album format is traditionally utilized for the purposes of narrative or melodic sequence, many well-known artists are now breaking the boundaries of music formatting.

"Drake releases playlists now. Beyonce makes visuals. Chance redefined the mixtape. Frank Ocean is releasing 45-minute music videos" (Seydel, 2017). These innovations definitely attract more attention from consumers and can promote an artist even further in their career. At the same time, these artists depend largely on the way algorithms process user engagement with their content through plays, skips, likes, dislikes, etc. This shift in music formatting has also changed the means that measure an artist's success, which not only includes album sales anymore, but also streaming numbers and digital album sales. In this new framework, achieving 1,500 streams on a single song is considered an "album sale," making it possible for an album to go Gold or Platinum purely based off of the success of that one song (Seydel, 2017). The importance of album-equivalent units have not only transformed the way music is marketed and released, but have heightened the stakes for artists to make it on to a mega-playlist and appear in user recommendations. 


\section{MUSIC RECOMMENDATION ALGORITHMS}

Rather than focusing on creativity and pushing the boundaries of the typical song format, artists are now more focused on "beating the skip rate", through the release of 1-2 minute singles that draw on simple, yet catchy, hooks and choruses that hit more quickly and with a greater impact. Many artists have purposefully produced longer albums with shorter songs in order to generate more streams and dominate the charts. "Ed Sheeran's new album $\div$ (Divide) is 16 songs long and only available as a deluxe edition (the standard version is shorter) on major streaming platforms, The Weeknd's Starboy is 18, DJ Khaled's new No. 1 Grateful is 22 tracks long and Drake's More Life playlist project features just as many tunes” (McIntyre, 2017). In fact, Drake's More Life playlist broke a Spotify record, achieving 76,355,041 streams on the first day of release, and 250 million streams between Apple Music and Spotify in the first 2.5 days. Additionally, this playlist offers the user "a 20-song sprawl of genres encompassing Hip-Hop, Trap, R\&B, Grime, Gospel, Dancehall, Tropical House and Afrobeat”, underscoring the potential flexibility and scope of music as cultural products (Meier \& Manzerolle, 2018). When artists release playlists or albums of this length, it is likely that their fans will stream all of the songs released. Not only will the number of plays increase compared to a shorter album, but it is likely for fans to hit replay more often to find the songs that they like the most. "It only takes just over 68 people to listen to Drake's new release to count as one "sale," whereas a 10-song collection would require 150 people to stick with it all the way through (McIntyre, 2017). The artists that are smart enough to manipulate the streaming algorithm in this way are earning No. 1 on the charts as well as breaking previous records. Nevertheless, this standardized formula for success is yielding a homogeneous culture, targeted at mass appeal rather than individual creativity and diversity. 


\section{MUSIC RECOMMENDATION ALGORITHMS}

Although algorithmic manipulation and standardization are fairly new concepts for the streaming industry to tackle, the radio industry has wrestled with similar issues for many, many years. One radio programmer rationalizes that "because we are BROADCASTERS trying to be MASS APPEAL, this explains why songs are repeated so often (a familiar and popular song has the best chance of keeping most of the listeners)" (Snickars, 2017, p. 195). Radio programmers defend this notion with studies that conclude, "people will say they want new music and new music discovery but we watch data that says listeners choose familiarity almost $100 \%$ of the time" (Snickars, 2017, p. 195). Though streaming platforms have developed these features for users to further explore artists and genres that are unknown to them, the notion of mass appeal, getting the most streams and the least skips, have consumed the minds of many artists and in turn, stunted the amount of risk-taking and creativity within music production.

\section{More of the Same}

Many streaming platform users have never even heard of recommendation algorithms, let alone know how they function. In fact, many users feel that they, themselves, play an active role in their music discovery experience and have full control over the content presented to them. "Due to the lack of transparency in how recommendations and 'discoveries' are presented, it is often not clear that these are promotional messages; rather they seem like grassroots discoveries based on a user's previous listening habits and patterns" (Morris \& Powers, 2015). This can be misleading for users as they expect and are made to feel like their favorite playlists and "personalized" recommendations echo their own tastes and interests rather than the interests of a commercial economy.

It is important for users to first recognize that a hierarchy of genres exists. Most often, "pop music" and "top hits" tend to be the first genres listed while the more niche and culturally 


\section{MUSIC RECOMMENDATION ALGORITHMS}

diverse genres like 'K-Pop', 'African', and 'Reggae' tend to be towards the end of genre lists on most of the top streaming platforms. SoundCloud, however, orders their genres alphabetically, which can pose its own hierarchical issues. Just as a person is more likely to click on the first link that appears after a Google search, a person would be more likely to choose one of the first playlists listed if they didn't have a genre already in mind. Music journalist, Scott Timberg argues that "Spotify is making you boring...With all the songs at our fingertips, we're exposed to very few, thanks to how digital recommendations work" (Timberg, 2016). Product Manager at Spotify, Matthew Ogle, advocates that their recommendation algorithms can connect "the smallest, strangest musician in the world" to the only "20 people in the world" that will dig their music (Pasick 2015); however, in order for the musician to share their existence with these 20 people, the recommendation algorithm needs to have a sense of these peoples' listening history and enough of this listening data for the algorithm to detect patterns between listeners. Not only is it unlikely that these 20 people have enough listening data in common for the algorithm to collaboratively filter the same artist recommendations to each person, but it is also unlikely for the algorithm to detect and prioritize the "smallest, strangest musician in the world" within its database amongst bigger, more popular artists.

Algorithms influence listener habits "whether driving your streaming playlists, your Amazon recommendations, or suggestions on iTunes...they are about driving you closer and closer to what you already know." (Timberg, 2016). Because collaborative filtering relies on the listening behavior of similar others, it is more likely for users to generate recommendations that fall within an infinite loop of the same artists and songs. This cyclical behavior may not be exclusively due to the mass appeal of familiarity, but also the implicit sequencing of songs within playlists, hierarchy of genres, and other promotional techniques embedded into the 


\section{MUSIC RECOMMENDATION ALGORITHMS}

interface of these platforms. It is ultimately up to the users themselves to find these lesser-known artists in order to influence their own recommendations as well as others with similar taste profiles. "Rather than providing a space for exploration it is possible that users can become trapped in these kind of "filter bubbles," where instead of guiding users "toward what you want to listen to, they direct you toward slight variations of what you're already consuming" (Timberg, 2016). Although algorithms ultimately learn from user behavior and listening patterns, it is important to recognize that platform developers and curators have the power and ability to drive users towards "the same" through the organization and design of content.

\section{Economic Influence}

In order to understand the way recommendation algorithms function within streaming platforms, it is also important to recognize that platforms are businesses, meaning that music recommendation has the potential to be motivated by capitalist interests. Not only have music streaming platforms like Spotify and SoundCloud built their business models off of the accumulation of user-generated data, but all-encompassing tech giants like Google, Facebook, Apple, and Amazon are also founded on the notion of "platform capitalism," as they provide both the hardware and software for others to operate on. These high performing technology companies interact with larger ecosystem lock-ins, providing a multitude of services for their allin users to take advantage of. For example, corporations like Apple and Google have access to a massive database of consumers from the various services they control and can use this customer base to their advantage when promoting and designing their music streaming services. Platforms are unique in that they are "far more than internet companies or tech companies, since they can operate anywhere, wherever digital interaction takes place" (Srnicek, p.44) and on any chosen device. This kind of media convergence makes it possible to listen to music streaming services 


\section{MUSIC RECOMMENDATION ALGORITHMS}

anywhere and everywhere you go. As platforms continually expand into markets worldwide, more and more users are able to participate in culture and connect with one another from almost any geographical location.

Although many platforms incentivize users to contribute to music culture through paid subscriptions or other monetization strategies, streaming platforms also rely heavily on the collection and selling of user data to advertising agencies. Platforms strive to accumulate the most users in order to collect the most representative and detailed data possible. This idea is known as "network effects," which suggests that the more users a platform has, the more valuable a platform becomes for everyone else. In music streaming, the platform that accumulates the most users has access to more data, which the recommendation algorithm can then use to compare more taste profiles with one another. The more information that can be collaboratively filtered, the better the algorithm will be, and the happier users are with their recommendations.

Although this business model can enhance the way recommendation algorithms process data, it can be argued that "this generates a cycle whereby more users beget more users, which leads to platforms having a natural tendency towards monopolization” (Srnicek, p. 45). Many streaming platforms not only take on the role of "a distribution outlet," but also that of a "promotional intermediary" (Morris \& Powers, 2015). This combination has the potential to be problematic, especially when the role of the promotional intermediary is commercially driven. “Spotify's 'programmatic advertising' business, under which media buying is automated, has not only enabled the company to 'attempt to maximize ad sales in a short time', but also to 'sell the idea of "music streaming behavior as a new currency for advertisers" to the venture capital investment community"” (Meier \& Manzerolle, 2018). Although traditional retailers have long 


\section{MUSIC RECOMMENDATION ALGORITHMS}

done in-store promotions for new releases or hosted events to coincide with local concerts, integrating promotional materials directly into the playback interface makes these efforts far more direct and immediate.

Many listeners have become reliant on in-house curated playlists to not only cater to their own personal tastes, but to also shape their future tastes. These listeners become malleable to the discretion of human curators and algorithms, which construct social and economic value within these playlists. Artists aspire to appear on playlists, like Spotify's 'RapCaviar' or Apple Music's “Today's Hits," because they have millions of followers and streams. While being placed on a popular playlist can be critical for an artist's success, the order in which they appear on this playlist is highly significant. It is important to mention that the artists that tend to be placed towards the top of these playlists also tend to be the more familiar, "popular" artists, and typically drive sales through commercial promotion. One source suggests that "the record labels have created a cottage industry out of promoting to these playlists as aggressively as possible, and some have pointed to their close relationship with the streaming services as the reincarnation of payola" (Ugwu, 2016). However, while record labels do have greater access to streaming distribution, most streaming platforms, excluding SoundCloud, have set up their business to exclude lesser-known, independent artists from distributing their content and rely on record labels to consistently deliver hits. For instance, Spotify has only recently allowed several hundred independent artists within the U.S. to join the Spotify for Artists program, out of the nearly 2 million artists they provide for. Streaming service employees have compared their tightly bound relationship with major record labels to that of the music press, saying that "while they receive a wide variety of regular promotional communications, there are strict policies in place to preserve editorial independence" (Ugwu, 2016). Most curators are left to their own 


\section{MUSIC RECOMMENDATION ALGORITHMS}

devices to determine the placement of specific artists or records. "More than simply being part of a field of distributed subjectivity, streaming platforms distribute subjectivity" (Flynn, 2016). Spotify, for instance, tends to give extra weight to playlists with more followers as well as the company's own curated playlists (Pasick, 2015). Not only does this prioritization reflect onto users' 'Discover Weekly' playlists, but Spotify also maximizes user data for the marketing and promotion of their in-house curated playlists. The image below is a promotional banner that appeared on the home page of my personal account, endorsing the Spotify playlists, "Winter Feels," "Pop Matters," "Hip Hop Made Me," and "Country Vibes.”

Figure 4: In-house Playlist Promotion

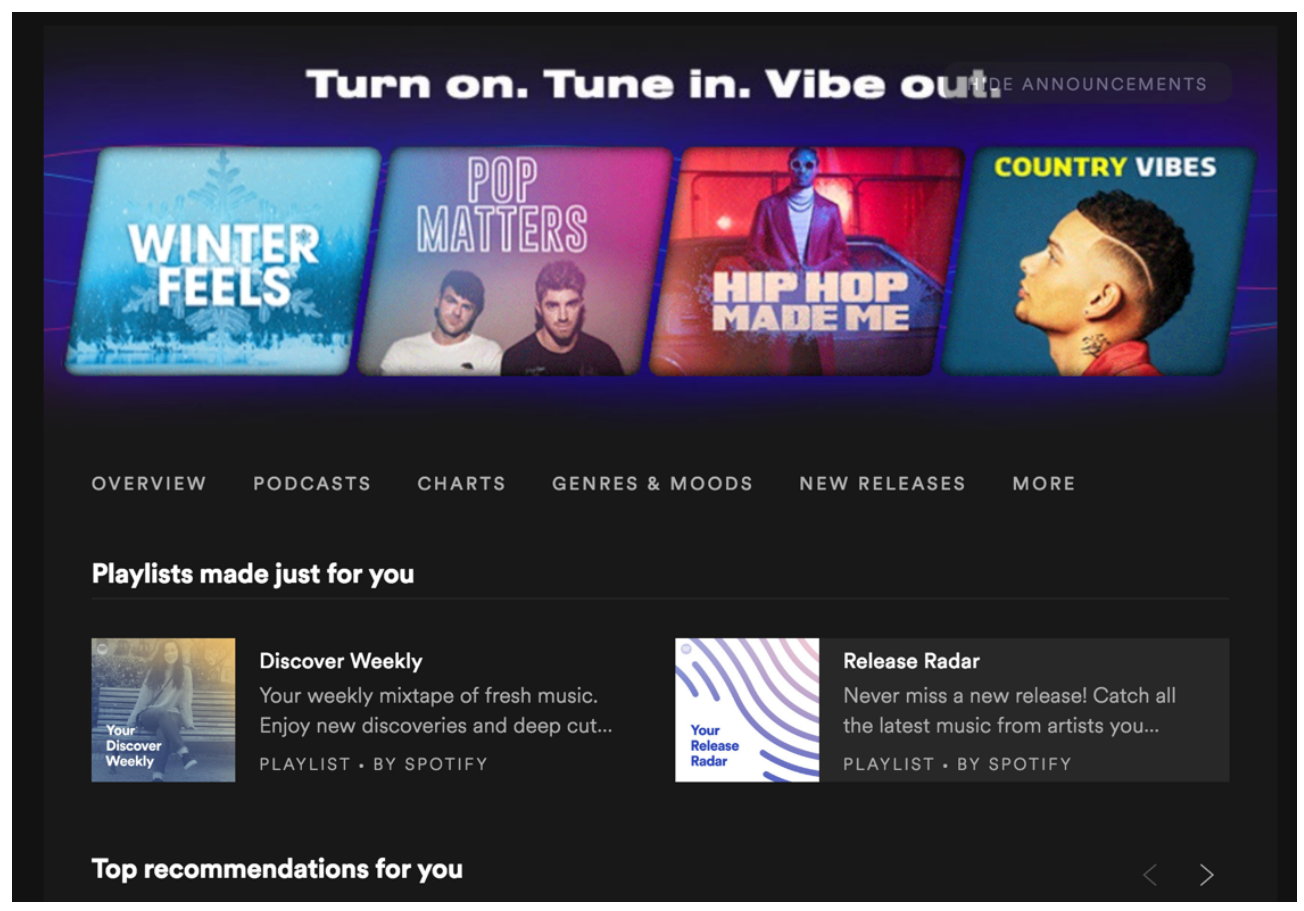

\section{(Spotify.com)}

Sponsored playlists are not solely characteristic of Spotify's business model. In fact, most streaming platforms will promote their playlists, and more prevalently use established brands to incorporate specific artists or music into their marketing messages. For example, a cruise line might sponsor a trendy 'Summer Vibes' playlist in their ad or a car company might market their 


\section{MUSIC RECOMMENDATION ALGORITHMS}

newest model using a feel-good 'Road Trip' playlist. Pandora's former Chief Scientist, Eric Bieschke, told New York Times that "the world of playing the perfect music to people and the world of playing perfect advertising to them are strikingly similar" (Singer, 2014). Rather than personalizing for specific individuals, advertisers target segments, creating a proxy for consumer categories constructed to fit the interests of specific brands. These categories tend to be only those that are economically meaningful. For example, in 2016, Spotify introduced 'Branded Moments,' a marketing plan promising to "identify - in real-time - what a listener is doing, and give brands an opportunity to own that moment," (Prey, 2018) organizing brands around six contents that cover all aspects of a person's day: 'chill time,' 'workout,' 'party,' 'dinner,' 'focus,' and 'sleep'. A popular alcohol brand, known as Bacardi, sponsored "party moments" through a campaign they called "We Are the Night." Bacardi identified "nine different "partytype people," which the company placed into categories with names like "brave shirts," "glow gals," and "last train sprinters" (Prey, 2018). By providing a scalable platform for advertisers to promote to a segmented market, streaming services "impose and normalize certain modes of contextualization at the expense of others" (Flynn, 2016). Specifically, digital listening has become a kind of tracking device, in that services are able to presume identity based on a user's preferences and then prescribe meanings to these preferences.

For example, in 2013, Pandora promoted the audience segment, "Hispanic listeners" and the sub-segment "Spanish-speaking listeners" to advertisers. Pandora cross-referenced zip codes collected from US census data with their own user registration data in order to categorize their listeners into this specific ethnic category. This strategy allowed for Pandora to compare the small sample of listeners who had already identified as Hispanic and look closely at the music most listened to by this sample in order to locate other Hispanic listeners across its entire user 


\section{MUSIC RECOMMENDATION ALGORITHMS}

base. This strategy conceptualized that "'Hispanic-ness" is performed into being through music listening behavior" (Prey, 2018). According to this method, if a user's listening behavior begins to change, so will their 'Hispanic-ness,' increasing the possibility for the listener to be assigned to another ethnic identity (Prey, 2018). This stereotypical categorization does not only project a cultural bias of what is and what is not considered "Hispanic," but can also provide unrepresentative data to both advertisers and recommendation systems. In turn, the data collected by advertisers has the potential to affect the way genre-specific playlists are constructed and ultimately shape the way specific cultures are perceived by society.

Many people who design and study streaming platforms have come to realize that "a good recommendation system should not rely on demographics because demographics discriminate" and "recommenders should only be interested in what consumers are interested in" (Prey, 2018). For instance, the identity of a traditional "classical music fan" should not be deduced based on a listener's age or socioeconomic status, but instead by a user's listening behavior. The listening data that is filtered into music recommendations doesn't reflect who we are but "who we might be - on our very proclivities and potentialities" (Amoore, 2011). The reflection of this projected self can affect the way we view ourselves as music listeners and predetermine who and what we listen to.

\section{Algorithm v Human}

Although people innately shift their musical identity and choose music according to their contextual state, there is still a sense of control that recommendation systems have over the music we find and listen to. Most users are unaware that an algorithm processes all of their listening data to produce song recommendations for them, let alone have an awareness that streaming platforms use their listening data to segment them into specific music identity 


\section{MUSIC RECOMMENDATION ALGORITHMS}

categories. The ambiguity of these algorithmic systems remains mostly invisible to the average user and is masked by aesthetically pleasing graphics and user-friendly language. This is why most users are extremely impressed with algorithmic curation. When their discovery playlists include a handful of accurate recommendations, users are often awestruck and feel that the platform really knows who they are and what they like, maybe even more than they know themselves sometimes. "Arguably, a lack of user awareness as to why the music they deliver in personalized playlists appears "really cool" is advantageous to the platforms" (Flynn, 2016). Because these algorithms are kept in a metaphorical "black box," most people are blind to the way they work and, by default, trust that this technology is objective and neutral as compared to human curation. However, it is important to recognize that this characterization can be deceptive.

Algorithms have backfired numerous times, including instances like Facebook's perpetuation of foreign interference in a superpower's election and Microsoft's AI-driven Twitter bot's assemblage of racist, sexist statements (Eder, 2018). Because the very concept of artificial intelligence is to embody traditionally human characteristics, it is inevitable for algorithms to project the biases that humans carry with them. Many media platforms that practice algorithmic recommendation have recently been criticized for what CEO of Facebook, Mark Zuckerberg, refers to as the spread of "sensationalist and provocative" content (Newton, 2019). In fact, YouTube has recently vowed to promote fewer videos that contain misinformation and conspiracy theories, attributing this problem to their default auto-play feature, which serves its users with new videos directly after a video finishes. "These rabbit holes often lead viewers to videos that have received lots of "engagement," including views, comments, and shares. More outrageous videos tend to perform well by these measures, and so they have been served up by the YouTube algorithm for years" (Newton, 2019). The prevalence of extreme content is not exclusive to social 


\section{MUSIC RECOMMENDATION ALGORITHMS}

media platforms, but can also be found on music streaming platforms. Specifically, a platform like SoundCloud, which lacks the gatekeeping abilities to fully control the content being distributed, allows for users to create and post whatever they want. While this freedom fosters open creativity among artists, that doesn't negate the fact that information is also more likely to be misinformed, inappropriate, or offensive. Unfortunately, algorithms are only able to process information in relation to stream accumulation rather than by the extremity of the content.

Human curation is often preferred due to the fact that logic and reasoning can potentially overshadow the feasibility and efficiency of algorithms. Specifically, Apple takes pride in their sole use of human-curated playlists, which are said to be designed by an "elite class of veteran music nerds" (Ugwu, 2016). Apple CEO Tim Cook takes a stab at algorithmic business models stating, "We worry about the humanity being drained out of music, about it becoming a bits-andbytes kind of world instead of the art and craft" (Miller, 2018). Despite the advanced technology within algorithms, many people still tend to consider a recommendation made by a trusted friend to be more reliable or valid. Algorithms are often perceived as mechanical or insensitive, while humans have a sort of social responsibility to connect with and satisfy listeners' needs. Though the identity of human curators tends to remain anonymous to the public, many people likely appreciate the sole fact that a human is creating recommendations for them and may even see

these curators in a similar light to a trusted friend, or even a personal DJ. Similar to the way a DJ is able to create transitions between songs when performing, human curators can curate specific tracks in a chronology that maintains the "flow" of a playlist. This is a quality that algorithms often lack.

While industry experience and the authentic human touch can provide for great music recommendation, any kind of human curation is going to be affected by personal taste. When 


\section{MUSIC RECOMMENDATION ALGORITHMS}

considering the economics of music streaming, it is vital for curators to remain as objective as possible when deciding which artists will make "the list". While Apple Music curators may be recognized for their keen taste and knowledge of music, it is important to look at the hands that create these influential playlists. Despite the promise to be "good shepherds and stewards" of

music and find the artists "that are going to mean the most and have the biggest impact" (Ugwu, 2016), subjectivity is inevitable in a business model that requires the promotion of a few over the rest. Putting the careers of so many artists into the hands of a few expert curators can be seen as a "form of elitism" that fosters both biased recommendations and misses on subtler trends (Kachkach, 2016).

\section{Cultural Influence}

Although algorithmic and human curation are largely affected by the people who control them, recommendation systems generally learn from and make decisions based on user behavior. The same way that human culture systematically includes and excludes people dependent on the rationality of certain groups, algorithms invite users to belong to distinct communities. "The design of algorithms and algorithmic procedures foster certain cultures, ideologies and identities" (Eriksson \& Johansson, 2017). When artists and sounds developed under the influence of Western ideology are prioritized in most playlists, realities of the world can be comprised, causing listeners to perceive culturally diverse music as less valuable. Recommendation algorithms are not only influenced by the subjective perceptions of listeners, but are also founded upon the intentions of their developers. For instance, Spotify's 'Discover Weekly' algorithm was created by a 36-year-old software engineer from New York, named Edward Newett. In some capacity, it is likely that the background and experiences of developers get projected into the application of these algorithms. Developers can "carry certain cultural categorizations" and either 


\section{MUSIC RECOMMENDATION ALGORITHMS}

intentionally or unintentionally provide a more detailed metadata for certain genres or certain geographical or cultural areas than others (Kuoppa, 2018, p. 72).

Typically, music created and distributed by the Western hemisphere has had a greater impact on music listened to by the rest of the world. "Top-ranked hits in the U.S. are still overwhelmingly focused on English-language and American-made songs-yet about 1 in 4 Spotify users around the world actively listens to artists from a culture (or country) different from their own" (Discover Hits, 2018). Based on this statistic, it seems imperative for recommendation algorithms to accurately represent a multi-cultural and multi-lingual selection in order to uphold the relevance and novelty expected of recommendations. As media gatekeepers, streaming platforms make an imprint on the way we construct the realities we live in and therefore, have a responsibility to represent cultures accurately and with integrity. This can include infusing more styles and languages into genre or country-specific playlists or even further promoting artists that have deep cultural roots in a specific genre.

Over the past few years, there actually has been a lot of improvement in terms of cultural diversification on streaming platforms. For example, Latin artists like Daddy Yankee, J Balvin, Ozuna, Bad Bunny, and Sofia Reyes have generated an international explosion of Latin music streamers and have actively progressed the genre into the top five album list in the U.S. (Acevedo, 2019). In fact, Latin music has surpassed country music in album listening, accounting for $9.4 \%$ over country's $8.7 \%$ stake (Acevedo, 2019). This surge in Latin music has not only affected the global streaming market, but has transformed the top charts of most Spanish-speaking countries from a mix of English and Spanish language content to almost exclusively Spanish language songs. Many streaming platforms have recognized the surge of 


\section{MUSIC RECOMMENDATION ALGORITHMS}

streamers listening to music beyond the scope of their own culture and have made efforts to address and diversify the music they recommend.

Most recently, Spotify curators have worked to broaden the platform's cultural influence with the launch of a playlist they call 'Global X.' This playlist specifically features "hot international and multicultural crossovers" (Discover Hits, 2018). Rocio Guerrero, Head of Global Cultures at Spotify, says that a crossover can be "a mix of different genres, a mix of languages, or it could be a song like 'Despacito Remix' that crosses over to the rest of the world." She further explains that "now people listen to 'Despacito' whether they are Latino or not. We are going to do the same with other cultures. Initially, you will see a lot of pop mainstream crossovers in the playlist, and while the point is to bring to the surface new sounds, cultures, and languages, we need to do so slowly but surely" (Discover Hits, 2018). Despite the confidence that Rocio and her team of international music experts have in their ability to integrate these international artists into the "popular," assimilating these sounds into the recommendations that algorithms produce will also require a shift in user behavior and listening patterns. While not impossible, it is likely to take a lot of time and strategic promotion in order to recalibrate these systems to assimilate more diversity into recommendations. It will be interesting to see how these cultural integrations affect the genres that penetrate into the mainstream global market over time and capture the attention of listeners the way Latin music recently has.

Although many countries' top hits have been consumed by Western-based music, most streaming platforms have also curated playlists dedicated to the music style of a specific country or nationality, such as Apple Music's "Best of Ghana” and "Brazillions: Excellent Tropicalia" or Spotify's "K-Pop" and "Arab" curations. However, the more familiar playlists and higher-ranked 


\section{MUSIC RECOMMENDATION ALGORITHMS}

genres are those that have been historically popular genres, typically including categories like "Pop," "Party," "Workout," "R\&B," and "Hip-hop." While a majority of users utilize the feature that allows them to curate and collaborate on their own playlists, nearly one-third of listening is actually done through in-house curated playlists (Kastrenakes, 2019). Today, playlists are considered to be "common currency" (Pasick, 2015), and are especially marketed this way on Spotify.

Most notably, Spotify's curated playlist, 'RapCaviar', has been considered "the most influential playlist in music," turning "mixtape rappers into megastars" (Marks, 2018). This playlist has become an instant path to success for a lot of rap artists. Placement on 'RapCaviar' is often seen as a form of verification that an artist has officially "made it". Unlike most curated playlists, Spotify has given 'RapCaviar' its own production staff and promotional campaigns, essentially treating this playlist as a sub-brand. "With editorial content, vertical music videos, a six-city concert tour, and over eight million subscribers, 'RapCaviar' has proven that the playlist can be far more than just a collection of songs updated once a week" (Singleton, 2017). Global programming head of hip-hop at Spotify, and curator of 'RapCaviar', Tuma Basa, explains that "Playlists make it easy to keep up with hip-hop...the same way that a newspaper made it easy for your dad to keep up with sports...because hip-hop moves fast. I do this for a living, and even for me it's a lot" (Marks, 2018). Arguably the most important and also risky component that Basa controls is the placement of songs and artists within the various rap playlists he curates. He takes these decisions extremely seriously, treating certain playlists, like 'Most Necessary' as a playlist to test out "not-quite-ready-for-prime-time jams" in order to decide if a song meets the expectations necessary to make an appearance on 'RapCaviar.' Often times, curators will "test" new and unfamiliar artists by placing them in subsidiary playlists to see what kind of reception 


\section{MUSIC RECOMMENDATION ALGORITHMS}

they receive. When referring to the promotion of Memphis rapper, Moneybagg Yo, from 'Most Necessary' to 'RapCaviar,' Basa says 'He's not on there yet...but it's like watching a college football player you know will make it to the NFL. He'll get there. I can tell” (Marks, 2017). With nearly 11.5 million followers, it is obvious that Rap fans rely heavily on the 'RapCaviar' playlist for music and artist discovery and expect Basa to consistently deliver hits.

When asked about the way Basa makes these critical decisions, he admits that while he utilizes "predictive skills — "gut, gut, and gut," he also turns to a trove of data that enables him to gauge a song's performance across the site: from how many times a song or artist has been searched for, to playlist-specific metrics such as percentage of people who skip the song (under 40 percent is desirable), percentage of saves to a user's own playlists and percentage of users who listen to more than 90 seconds of a song, known as completion rate," when he doubts his instincts (Marks, 2017). It is surprising that even the curation decisions of an experienced and esteemed human curator like Basa are somewhat shaped by algorithmically-derived data, posing the thought that algorithmic and human curation may regularly intermix within one another. Saavn's, Rishi Malhotra, says that when it comes to programming music, "you need machine and programmatic algorithms to scale; and you need humans to make it real" (Croll, 2015). While algorithms provide insight to human curators, human curators also affect the way algorithms function. Being that the music streamed is mostly derived from these human-curated playlists, the placement and sequencing of songs is an essential factor in shaping recommendation algorithms. CEO of Sony Music, Rob Stringer, indicates that the 'RapCaviar playlist' "has a fair degree of personal taste" and admits "I don't think the streaming platforms would ever say that it's purely about the algorithm. And we don't want it to be about the algorithm. Quite frankly, the same way as we also didn't want it to be, in the previous chapter, only radio producers 


\section{MUSIC RECOMMENDATION ALGORITHMS}

deciding what was good to go on the radios because there's obviously plenty of examples in the past where radio stations said, "That isn't a hit" and then it turned out to be a really big hit" (The Media Show, 2018). Finding a balance between the influence of human and algorithmic curation seems to be both the greatest challenge and the utmost necessity to providing successful music recommendation.

In recent news, Spotify has been projected to transform some of their previously humancurated playlists into personalized algorithmically-driven playlists, similar to the design of 'Discover Weekly'. Spotify reports that human editors will continue to pick and choose which songs fit into a certain playlist; however, every user's playlist will include a different combination of these songs depending on their own taste profile. Not only will this feature allow for more diversification within genres, but it will also allow for the exposure of more artists and songs. Spotify says that with more personalized playlists, "the number of artists on each playlist goes up by 30 percent and the number of tracks goes up by 35 percent" (Kastrenakes, 2019). Additionally, when an artist's song is added to a personalized playlist, they will be able to share a custom link with their fans, which fans can then access to place that artist's song at the top of their playlist. It seems inevitable that streaming platforms will continue down this path of personalization. Diversification within genre categories and artist exposure are optimal under these conditions and will likely generate a wider variety of novel and relevant recommendations expected from users.

\section{The Walk-Through Method: 'Discover Weekly' and 'SoundCloud Weekly'}

To better understand if music discovery playlists accurately reflect a user's taste profile comprised of an equal representation of each genre category, I conducted my own experiment using the "walk-through method," also known as "an approach to the study of apps." 


\section{MUSIC RECOMMENDATION ALGORITHMS}

The Walk-Through Method:

"This method is grounded in a combination of science and technology studies with cultural studies, through which researchers can perform a critical analysis of a given app. The method involves establishing an app's environment of expected use by identifying and describing its vision, operating model and modes of governance. It then deploys a walkthrough technique to systematically and forensically step through the various stages of app registration and entry, everyday use and discontinuation of use. The walkthrough method establishes a foundational corpus of data upon which can be built a more detailed analysis of an app's intended purpose, embedded cultural meanings and implied ideal users and uses" (Light, Burgess \& Duguay, 2016).

The walk-through method is significant for this body of research because it allows for the analyzation of a step-by-step process, from new user registration to listening behavior to song recommendation. In order to fully grasp the experience of a new music streaming user and explore the consequences of each action made by this user, I created new user accounts for the free versions of both SoundCloud and the Spotify application. I chose SoundCloud and Spotify specifically because these two platforms are the only services to provide algorithmically curated playlists that are solely dedicated to music discovery. Although other platforms have similar playlists devoted to new releases, genre-specific selections, and social network-based recommendations, 'SoundCloud Weekly' and 'Discover Weekly' are expected to provide novel and relevant recommendations. Because each action made by a user greatly affects the output of algorithmic recommendations, it was crucial to take screen-shots of not only each action made during the listening period, but also the organization of the interface, including the order of 


\section{MUSIC RECOMMENDATION ALGORITHMS}

genres, the sequencing of songs, the promoted advertisements, and most importantly, the lists of recommended songs within both the 'SoundCloud Weekly' and 'Discover Weekly' playlists.

\section{Methodology}

To begin the walk-through of both platforms, I first needed to ensure that my identity as a user was as concealed as possible and thus, make it impossible for the computer to retrieve any data regarding my geographical location, demographics, or other defining characteristics that could potentially categorize me into an identity "filter bubble". In order to conduct this experiment as objectively as possible, I not only used a private browser on my computer, but also created fake email accounts for my new user accounts using a disposable temporary email generator. When registering, I was required to provide an age, gender, and username for each platform. I chose to enter the age " 35 " being that it seemed like a neutral age within the traditionally young adult age range of music streamers. For gender, I entered the option that said "Prefer not to say" and for username I used the name, "User." While any choices I made could potentially affect the recommendation algorithm, I believe that these choices were the least subjective options.

After creating a new profile for both SoundCloud and Spotify, I screen captured the initial interface that appears as soon as a new user is registered. Next, I screen captured the 'SoundCloud Weekly' and 'Discover Weekly' playlist pages, which were, as expected, empty due to a lack of listening history. In order to generate enough data for the recommendation algorithm to create a list of 30 "discovery" songs, I chose to listen to the first song listed under each listed genre, within the first curated playlist listed. SoundCloud has 41 different genres available and Spotify has 42 different genres available. Some of the more basic genres like "Pop," "Country," "Classical," and "Latin" are common between the two platforms; however, 


\section{MUSIC RECOMMENDATION ALGORITHMS}

there are many genre categories that differ. Not only do categories differ, but the top songs listed under each category are strikingly different, meaning that each platform has a very distinctive perspective on the categorization of music into certain genres. These perceptive differences may affect the recommendations in each playlist; however, those details will be delineated later in the results section.

As mentioned previously, a song is generally considered "listened to" after playing it for 30 seconds. I not only made sure to listen to each selected song in its entirety, but I also favorite/liked each of the songs listened to so that the platform would perceive that I "liked" them and then translate these interests algorithmically. I screen captured each song as I listened in order to keep an organized record and I also took screenshots of numerous advertisements that appeared throughout my listening session. About two weeks after this experimental listening session, my ‘SoundCloud Weekly’ and ‘Discover Weekly’ playlists were available, each providing 30 song recommendations, spanning about an hour and 40 minutes worth of content. For each song listened to and song recommended, I recorded the genre of music, year of release, promotional messages, and whether the artist was independent or signed by a record label, and specifically for SoundCloud, the type of user the artist was (Pro Unlimited, Pro, or Free). By comparing the categories of the songs listened to versus those of the songs recommended, I hoped for the results to reveal which genres may be favored by each platform, the time period most common to recommendations given, and also whether record label contracts and/or premium access affect how often artists appear in these recommendations. Lastly, I hoped to reveal the ways that artist and playlist promotion is presented both obviously and discretely within the interface, to strategically draw a user's attention towards a specific artist or playlist. Once considering all of these significant factors, this experiment should observe and assess 


\section{MUSIC RECOMMENDATION ALGORITHMS}

patterns between SoundCloud and Spotify's recommendation algorithms. These results should ultimately discern the recommendation algorithm's ability to deliver "novel" and/or "relevant" selections as well as a representative sample of songs based on the given listening history.

\section{Results}

\section{Genre}

When testing the 41 different SoundCloud genres listened to against the genres appearing in my 'SoundCloud Weekly' playlist, it was obvious that one genre appeared much more often than others throughout the recommendations. This genre was 'Hip-hop \& Rap.' In fact, 83\% of songs recommended fell into this genre category, versus only $10 \%$ 'R\&B', $3 \%$ 'Pop', 3\% 'Trap,' and virtually none of any other genre category. Although these recommended songs have potential to fall under multiple genres, I only considered the main genre that these tracks were tagged with. The chart below (Figure 5) depicts the imbalance of genre representation, with 'Hip-hop \& Rap' at the peak of recommended genres.

Figure 5: SoundCloud Genre - Songs Listened To vs. SoundCloud Weekly Songs

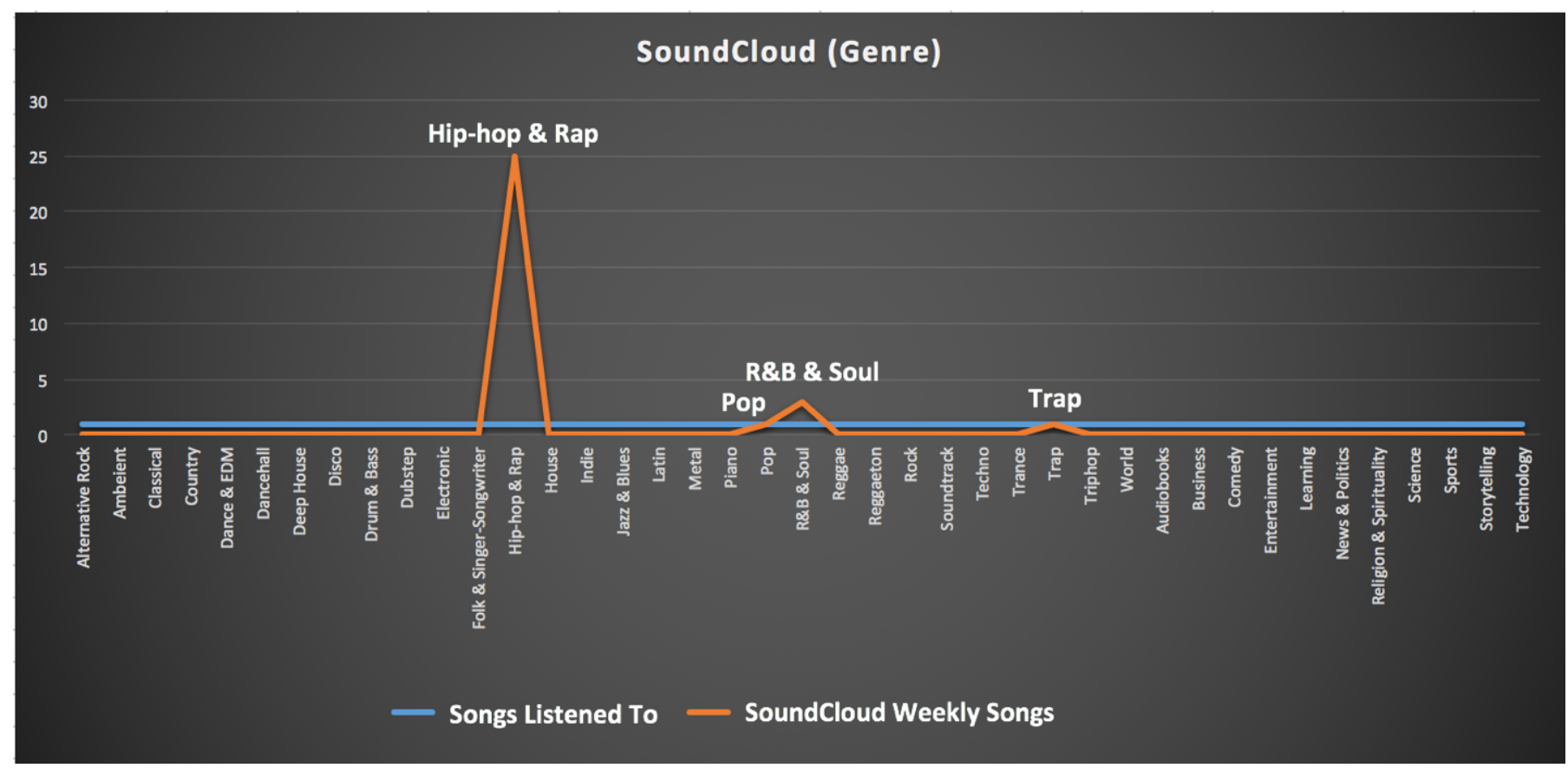




\section{MUSIC RECOMMENDATION ALGORITHMS}

Considering the platform's reputation and user base, it is not a total shock that Hip-hop and Rap would be the most common genre recommended. For one, SoundCloud is well-known for providing a platform for amateur Hip-hop and Rap artists to express themselves and has facilitated the emergence of a community of SoundCloud rappers. "The term "SoundCloud rap" is one of these things - a description so ubiquitous that it has come to feel like ad-agency shorthand, like "hipster" or "millennial"” (Battan, 2019). Many of these rap artists have surfaced into mainstream music and have developed into some of the most famous rappers of the modern era, including artists like Juice WRLD, XXXTentacion, Lil Uzi Vert, Post Malone, 21 Savage, Lil Pump, and many more. In fact, a majority of the artists listed appeared often throughout this experiment. Unlike any other genre, it seems that there is not only a demand from users for the rise of "grassroots" rappers, but also a demand from record labels to discover and cultivate the next generation of famous rappers.

Despite the recognition SoundCloud has for its Hip-hop and Rap categories, "mistagging" is also a possible cause for this misrepresentation of genres. By "mistagging" I am referring to the fact that a song may be placed under a certain genre algorithmically due to the way an artist tags their song, but it may not directly match the criteria expected of that genre. Different from Spotify’s hand-curated genre playlists that are categorized by "experts", SoundCloud's genre playlists are comprised of songs that have the most streams and are categorized on the basis of being "tagged." For example, when listening to the top song streamed under the genre "Country," I quickly noticed that the artists singing the song, BIG CHILD SUPPORT \& LIL ROCKSTAR, were clearly rappers, and that the song itself was purely a rap song; however, it was tagged "\#Country". To confirm my inquiries, there were many comments complaining about this discrepancy. One comment read "* Looks up Country music charts on 


\section{MUSIC RECOMMENDATION ALGORITHMS}

Soundcloud.* *Sees a rap song.* Soundcloud in a nutshell.” Throughout my SoundCloud listening session, I noticed this distortion of genres happening quite frequently and realized that many of the songs I was listening to were either clearly Rap songs or had potential to be categorized as Rap songs. It is extremely probable that within the 'SoundCloud Weekly' algorithm, Hip-hop and Rap consequently acquire more user data and can be recommended more often than other genres. In effect, SoundCloud users' listening identities will more commonly relate to one another through Hip-hop and Rap than any other genre. Additionally, natural language processors and convolutional neural networks are more likely to identify Rap-specific language and acoustics from the songs listened to and associate them with other similar Rap songs. The lack of user data across most other genres relates back to the "cold-start problem," where conditions are not yet optimal for the platform to provide the best, or most accurate results.

In terms of 'Spotify Weekly' recommendations (see Figure 6), 40\% of the songs were categorized as 'R\&B', $18 \%$ 'Soul', 16\% 'Pop', 7\% 'Hip-hop', 7\% 'Indie', 5\% Dance/EDM', and 2\% each for 'Funk', 'Afro', 'Folk \& Acoustic', 'Rock', and 'Country'. Although ' $R \& B$ ' is clearly the genre spanning the most recommendations, 'Discover Weekly' resulted in much more variety than 'SoundCloud Weekly.' In fact, there is likely even more variety than listed in the graph below being that Spotify does not directly tag each song with its genre category. In order to categorize each song, I had to do some of my own research by searching each artist and song both on the Internet and within the Spotify app, in order to see what kind of genre-specific playlists the song had been featured on and what the general consensus was online concerning genre placement. Additionally, the names of Spotify's genres are much broader than SoundCloud's, making it more difficult to label a song with a specific 


\section{MUSIC RECOMMENDATION ALGORITHMS}

genre or group of genres. For example, genres like 'Mood', 'Workout', 'Chill', 'Focus', 'Party' and 'Pop Culture' are difficult to detect among recommendations being that these categories are much more subjective to personal opinion and taste. However, it was much easier to detect the absence of more universally identifiable genres, including 'K-Pop', 'Arab', 'Desi', 'Classical', and 'Metal'. It seems like no coincidence that the genres missing in 'Discover Weekly' recommendations tended to be those that fall towards the bottom in Spotify's hierarchy of genres. The genres listed lower tend to receive less user attention and therefore, could affect the amount of user data amassed from a specific genre. Despite these inequities, Spotify’s community of listeners seem to be much more diverse in genre taste than SoundCloud's Hip-hop and Rap focused community, which is likely reflective of a more diverse playlist of discoverable music.

Figure 6: Spotify Genre - Songs Listened To vs. Discover Weekly Songs

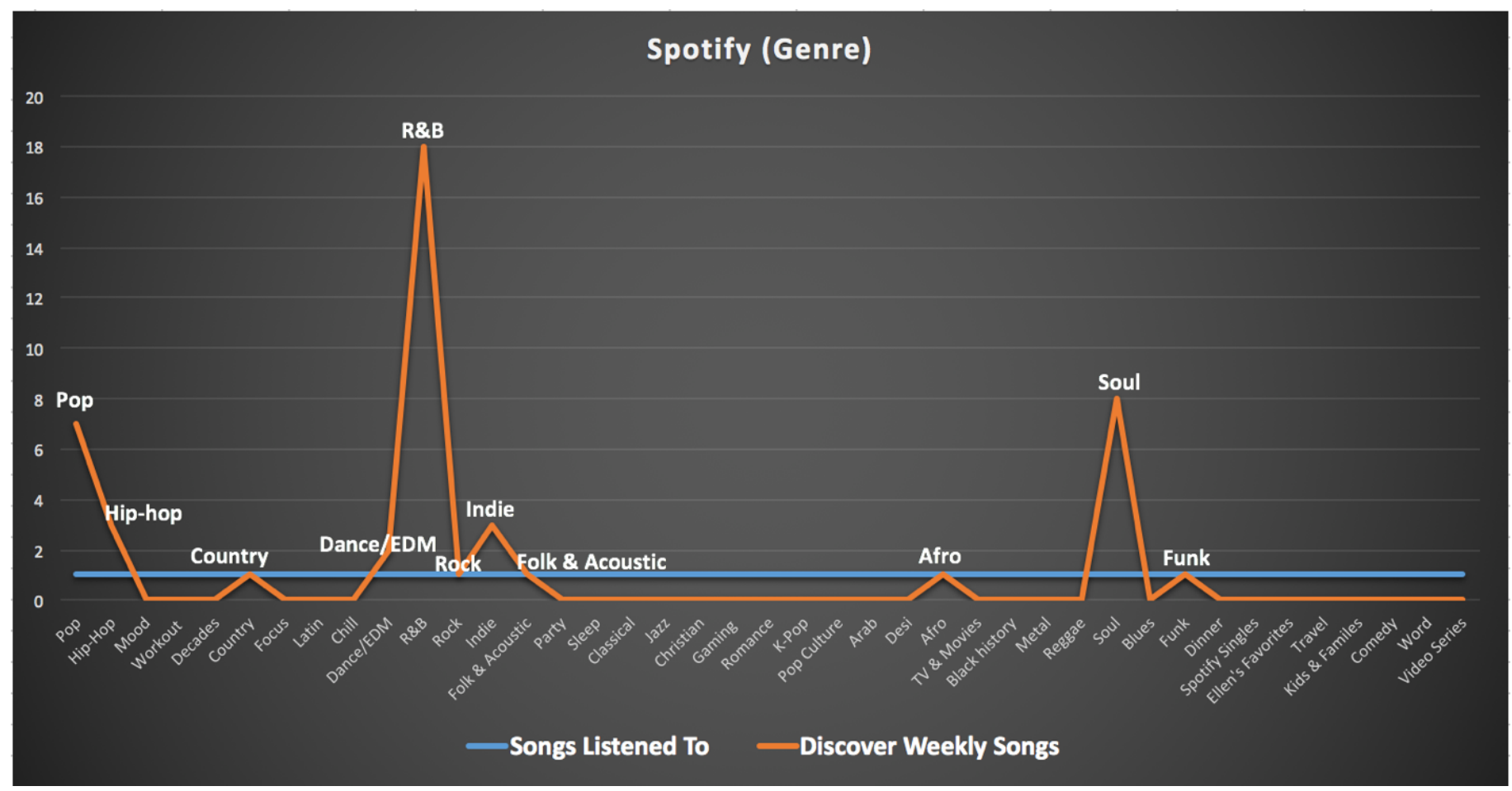




\section{MUSIC RECOMMENDATION ALGORITHMS}

According to Nielsen Music's 2017 report, R\&B and Hip-hop represented 24.5 percent of all music consumed in the U.S., replacing Rock, which claims 23\% (Caulfield, 2018). These percentages encompass the culmination of album sales, song downloads, and streams. Eight out of the ten most listened-to artists included hip-hop legends, Drake, with 4.8 million album equivalent units and Kendrick Lamar with 3.7 million (Ryan, 2018). Other standouts included Post Malone's Congratulations ranking among 2017's 10 best selling tracks and former “SoundCloud rapper," Lil Uzi Vert's viral hit, XO Tour Lif3, reaching 4x platinum. Nielsen also noted that the immense growth of R\&B and Hip-hop genres was "powered by a $72 \%$ increase in on-demand audio streaming" (Lynch, 2018). Streaming culture has given these genres the power to redefine cultural norms. Hip-hop, in particular, is unique in its ability to merge with social and political boundaries, including "fashion, language, education, censorship, and endorsements" (Pybis, 2018). Being that this genre is so dependent on one's own background and experience, artists of different nationalities, ethnicities, and religions have defined and cultivated their own Hip-hop cultures across the globe. Not only have Hip-hop and R\&B genres diversified with new classifications and sub-genres, but they have become a global sensation, transforming the way we typically define "popular" music. This shift in music consumption is vital in understanding the changing landscape of streaming platforms and the ways in which the growth of these genres will affect the kind of music that is produced, distributed, and consumed by the masses. It is obvious that despite the wide variation of genres listened to in this experiment, recommendation algorithms will continue to mirror the trends set by the global market.

\section{Release Year}

One of the first trends I noticed while listening to both the genre-specific songs and discovery playlist songs was that a majority of the content was comprised of music released over 


\section{MUSIC RECOMMENDATION ALGORITHMS}

the past two years, 2018 and 2019, with the rest of the tracks sporadically arranged throughout previous years. Although many streaming services provide playlists dedicated solely to new releases, including playlists like 'Release Radar' or 'New Music Friday', discovery playlists imply that recommendations are not necessarily time-sensitive and can include music spanning the entirety of its historical archive. The bar graphs below show that all songs listened to on SoundCloud were distributed over a period of time from 2008 to 2019 (see Figure 7) and all songs on Spotify ranged from 1950 to 2019 (see Figure 8). In both cases, the genre-based songs were much more diverse in time frame than were the recommended songs on the discovery playlists. Even so, the 2018 and 2019 genre-based selections on SoundCloud make up over 50\% of songs listened to and on Spotify, nearly 80\%. On Spotify, the track released in 1950 was actually a piece of spoken word, and not a selection of music, and the track released in 1976 was a Stevie Wonder song from one of Spotify's lesser-known, obscure playlists curated by Ellen DeGeneres. Considering that the 'Word' and 'Ellen' playlists are listed last and $5^{\text {th }}$ to last within the order of genres, it seems unlikely for them to accumulate a lot of user data and it is safe to say that these data points can be considered outliers. Although the genre-specific tracks are comprised largely of newly released music, both 'SoundCloud Weekly' and 'Discover Weekly' recommendations are overwhelmingly skewed towards newly released music. In fact, over $75 \%$ of 'SoundCloud Weekly's' playlist and 100\% of "Discover Weekly's' playlists are made up of songs released only between the years of 2018 and 2019. Being that the discovery playlist songs are much more concentrated in 2018 than they are in 2019, this may indicate signs of a "coldstart problem" within the algorithm, where newer songs have attracted less user mobilization, creating less user data for the algorithm to process. 


\section{MUSIC RECOMMENDATION ALGORITHMS}

Figure 7: SoundCloud Release Year - Songs Listened To vs. SoundCloud Weekly Songs



Figure 8: Spotify Release Year - Songs Listened To vs. Discover Weekly Songs

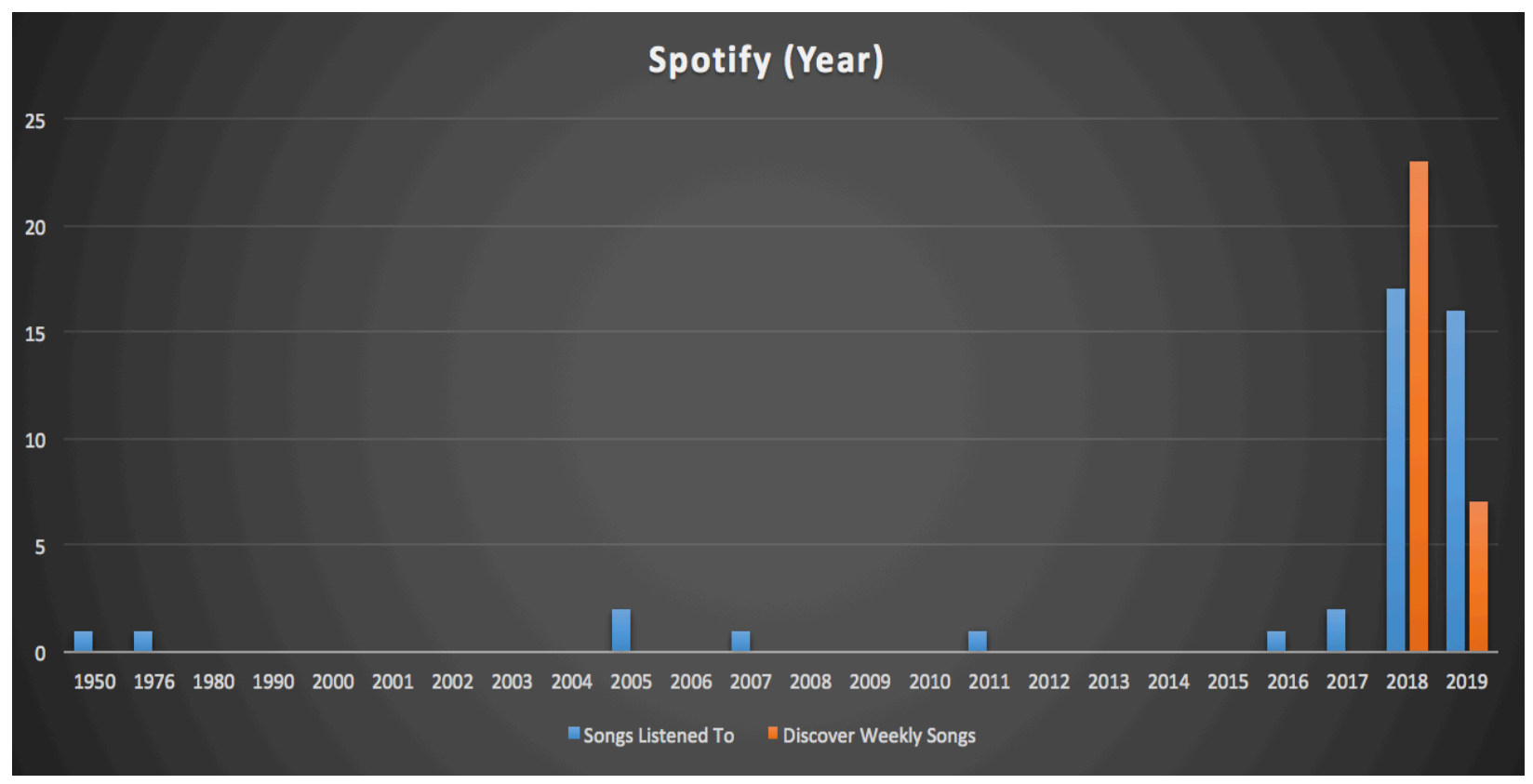

The most obvious deduction from this data, however, is that most playlists offered to users don't include music that dates back earlier than the $21^{\text {st }}$ century. While producing "novel" recommendations may be attributed with music that is new, "novel" can also mean innovative or unique, which aren't necessarily stamped with a time. Although new music is likely to receive 


\section{MUSIC RECOMMENDATION ALGORITHMS}

more attention from users due to promotion and fan interest, streaming platforms have worked over the past few years to re-master and digitalize tons of older physical recordings from records, CDs, cassettes, and live recordings. For example, just a few years ago, The Beatles' original 13 albums, along with a few post-1970 compilations, were released on most streaming platforms. Giles Martin, son of Beatles producer Sir George Martin, exclaims that "The Beatles needed to be on all streaming services if they were to reach out to a new generation of music fans" (Titlow, 2018). In order to maintain relevance, artists of the pre-digitalized era have realized that it essential to conform to new formats of music listening and digital distribution. Not only should streaming services continue to encourage the addition of older catalogs, but they should also aim to incorporate them into genres that aren't solely dedicated to throwbacks or specific decades. It's important for listeners, especially those who are younger, to have a sense of the sounds and icons that have inspired most of the music we enjoy today. Recommendations would definitely be broadened historically and culturally if older catalogs were made more accessible and apparent to users. A good start would be by intermixing older songs into genre-curated playlists, which would hopefully be reflected into users' music recommendations.

\section{Artist Status}

Although genre and release year are vital factors to consider when analyzing music recommendations, one of the most influential factors is an artist's status. SoundCloud, for instance, has Premier subscription tiers, Pro and Pro Unlimited. According to the results seen in Figure 9, 57\% of recommended artists were Pro Unlimited users, 13\% were Pro users, and 30\% free users. Pro Unlimited users pay the most per month, and in turn, receive the most benefits, including scheduled releases, full stats, full embed controls, access to SoundCloud Go+, and 


\section{MUSIC RECOMMENDATION ALGORITHMS}

unlimited upload time (SoundCloud Premier, n.d.). These benefits can be extremely significant in exposing an artist's music to a large audience.

Figure 9: SoundCloud User Type - Pro Unlimited, Pro, and Free Users

\section{SoundCloud User Type}

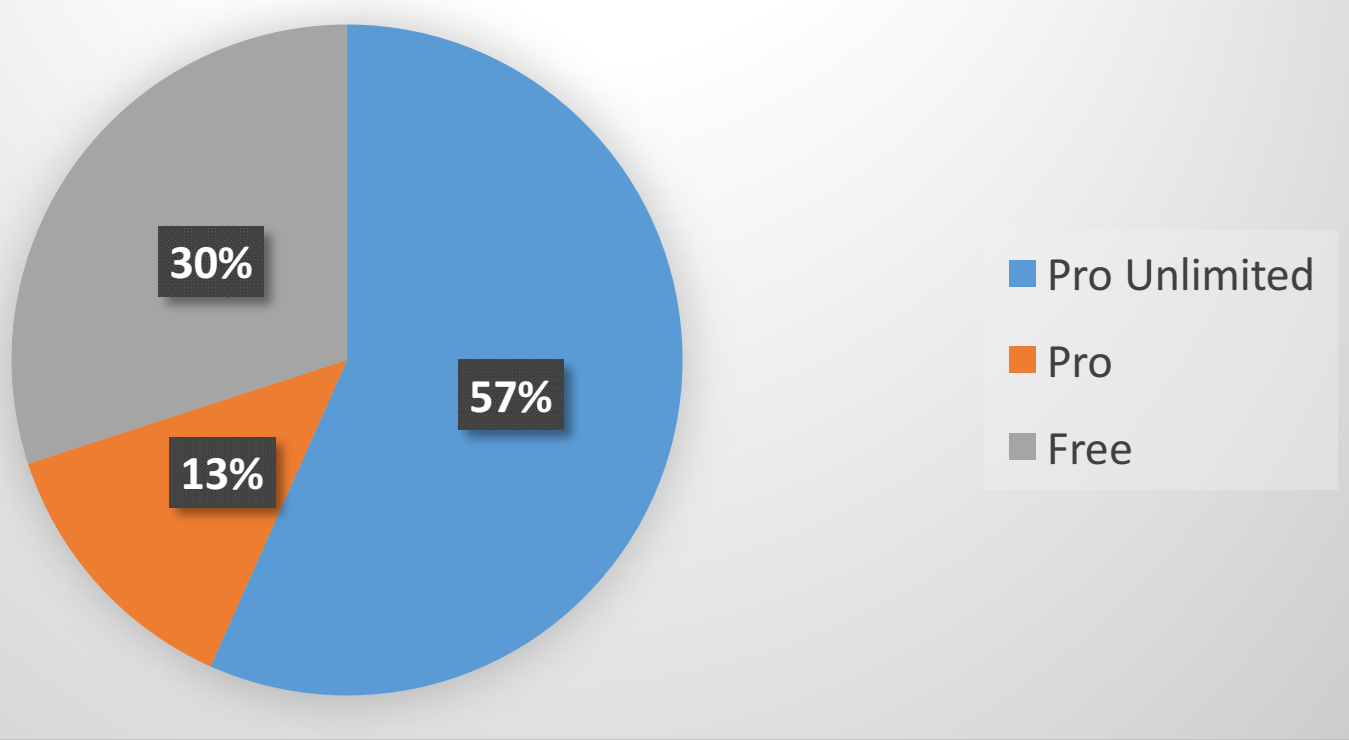

As illustrated in the pie charts below, 39\% of genre-based songs on SoundCloud and $57 \%$ of

'SoundCloud Weekly' songs were produced by record label artists.

Figure 10: SoundCloud Artists - Proportion of Independent vs. Record Label Artists

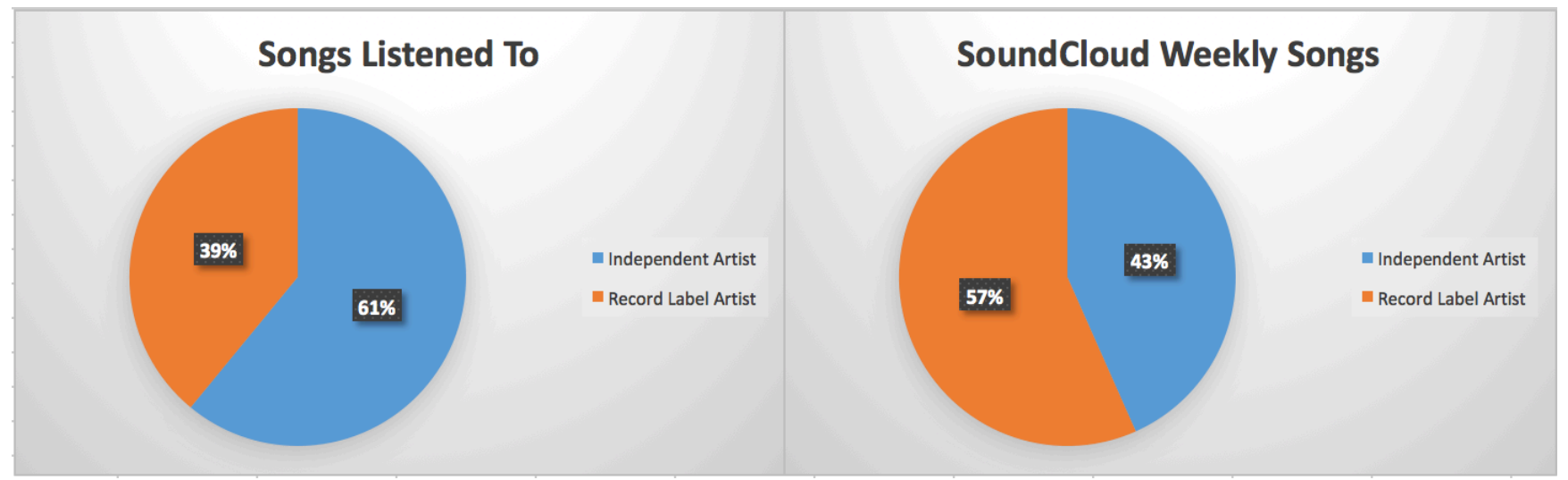




\section{MUSIC RECOMMENDATION ALGORITHMS}

Although independent artists seem to frequently surface into genre-specific playlists on SoundCloud, it seems that these artists are slightly disadvantaged when it comes to being discovered in the 'SoundCloud Weekly' playlist. On the other hand, there is no representation of Spotify's artist-type distribution because all of the artists listened to were record label artists. This may be because it wasn't until recently, in September of 2018, that Spotify began to allow a select group of independent artists to upload their music to their platform, without requiring a contracted distributor to handle licensing, distribution, or streaming royalties. "According to Billboard, this feature is only open to a few hundred U.S.-based independent artists," (Payne, 2018) out of the nearly 2 million artists that Spotify houses. This shutout of independent artists makes it virtually impossible for independent artists to surface into curated playlists, let alone 'Discover Weekly' playlists. This kind of exclusive gatekeeping diminishes independent artists' abilities to distribute original content as well as interact and collaborate with commercial music in the same way that SoundCloud enables them to. While this kind of digital rights management can be beneficial for shutting out illegal reproductions of music, there should be another way for more independent artists to share their creativity on such a large platform in a controlled and regulated environment. Inviting unsigned artists to Spotify's platform is just the start to "nurturing the sounds that may one day spring out of it" (Hogan, 2018). Just as "YouTube introduced the world to a new generation of teen heartthrobs" and "Vine helped spread viral dances," Spotify has the clout and scale to give cultural and profitable value to music innovation all over the world.

Despite SoundCloud's inclusive business model, independent SoundCloud artists actually showed an $18 \%$ decrease from genre-specific songs to 'SoundCloud Weekly' songs. As expected though, almost all Pro Unlimited users featured on this 'SoundCloud Weekly' playlist are signed 


\section{MUSIC RECOMMENDATION ALGORITHMS}

to major record labels, and every free user featured was unsigned. Most free users seemed to be the kind of "grassroots" musicians that SoundCloud proudly champions in the branding of their platform. Many of these free users seem to specialize and are passionate about covering, remixing, and mashing up songs that are already popular top hits, rather than creating their own original content. For example, an artist by the name of Vicidex mentions that in his rendition of Juice WRLD's "Wasted” ft. Lil Uzi Vert, he "sped up the track by 1.1x." Like many remixers on SoundCloud do, Vicidex also mentions that there is "no copyright infringement intended," however, it is definitely possible for Juice WRLD's representation to issue a takedown notice if they feel their artist's content is not being used under the terms of fair use. Another artist by the name of XOJXCOB315 writes "this is for cover purposes only" in his I'm Not Scared "Scared of the Dark” Cover/Remix and artist, LIOHN, captions his remix of Red Roses by Lil Skies by stating "It's such a banger already, but I just had this urge to add that little digital spice to it." While this collaborative culture among artists is encouraged by the SoundCloud community, it is not always supported legally. It seems vital, however, to the success of free user/non-record label artists to be able to borrow, re-assemble, and experiment with these units of cultural information in order to gain exposure.

Surprisingly, there weren't many independent, free SoundCloud users producing original content. This may be because an independent artist likely does not have the loyalty of many fans yet and it seems that listeners are drawn more towards renditions of already known and liked songs, or towards the "familiar." As a newer, independent artist, it is wise to gain a fan base through this kind of repurposing of music, either by sampling or covering, and later build up to the release of original content. By removing the distribution gatekeeper, not only does SoundCloud provide for collaborative production to help lesser-known artists express themselves 


\section{MUSIC RECOMMENDATION ALGORITHMS}

on a massive platform, but they also foster a broader environment of new and meaningful content for users to discover and evaluate.

\section{Content Promotion}

Another fundamental aspect of streaming that affects an artist's exposure and relevance within algorithmically-curated discovery playlists is in-house promotion. Advertisements are an essential marketing tool for drawing users' attention to artists and playlists. Throughout the new user listening experience, it was obvious that many of the advertisements built into the free versions of SoundCloud and Spotify promoted the premium versions of each platform, which can be seen in Figures 11-14. The first advertisement is a SoundCloud promotion advertising its full access, ad-free, subscription version, SoundCloud Go (see Figure 11). This ad is captioned, "The Next Big Artist Is Already Streaming Here," speaking not only to the amateur culture that SoundCloud appeals to, but also poses a challenge to users to discover the "Next Big Artist". The person pictured in this advertisement is most likely meant to signify a SoundCloud user who has possibly found the "Next Big Artist." Many artists have started from nothing on SoundCloud and used this platform to gain exposure. This exposure has led to the signing of many amateur artists to major record labels, which have the tools and resources to further develop the potential success of artists. SoundCloud utilizes this ability to connect artists with a larger audience to their advantage when marketing their brand.

The Spotify advertisements in Figures 12-14 also promote their Premium subscription service to new users using their platform free of charge. Spotify also often promotes their own curated playlists, including one they call 'Bedroom Jams' (seen in Figure 12). Playlists are often promoted either in a stagnant ad, like this one, or in video format, which usually feature popular artists that are likely to appear in these playlists or artists that typically represent that genre. The 


\section{MUSIC RECOMMENDATION ALGORITHMS}

advertisement in Figure 13 was the header of Spotify's home page during the new user experiment, promoting the new release of SG Lewis and Clairo's single 'Throwaway'. These socalled "announcements" are not exclusive to singles, but also frequently promote EPs, albums, and various artists. The last advertisement pictured is a screenshot from a commercial-like advertisement that depicts the collaboration between famous hip-hop artist, Jayden Smith, and the popular shoe brand, New Balance. As mentioned previously, brands often team up with popular artists or playlists and incorporate certain ideals associated with various artists and genres into their marketing messages. Unlike the advertisements that appeared on SoundCloud, Spotify's advertisements frequently feature well-known, popular artists. This dissimilarity gives insight to the artists each platform intends to cater to. Whether reality matches each platform's intentions or not, the promotion of an artist on a streaming platform has the capability of directing audience flows towards that artist and therefore, should feature a diversity of artists in order to broaden the listening trends that get processed by recommendation algorithms.

Figure 11: SoundCloud In-House Advertising

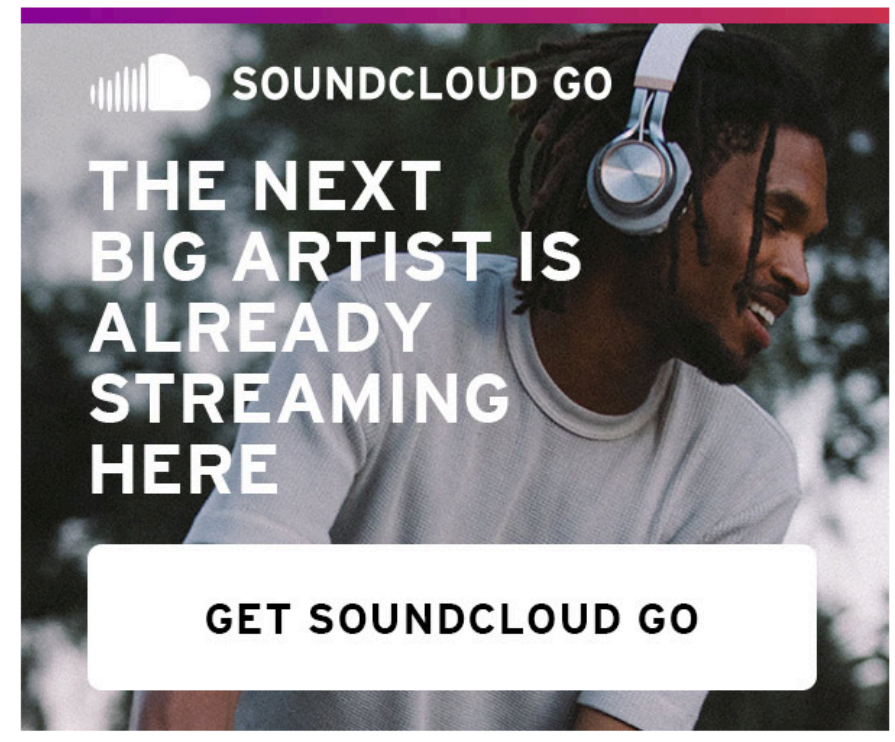

(Soundcloud.com) 
MUSIC RECOMMENDATION ALGORITHMS

Figure 12: Spotify Playlist Promotion

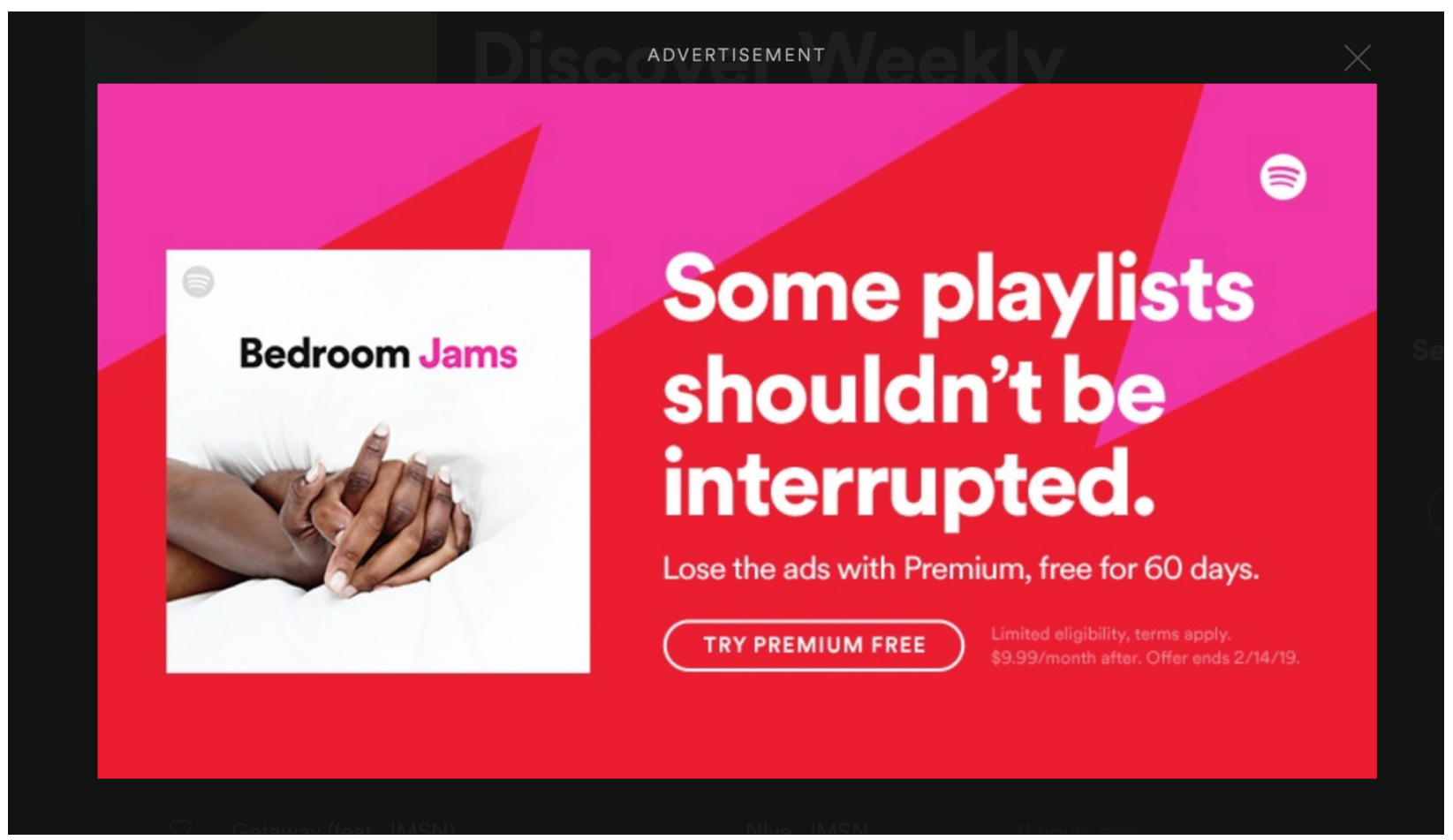

(Spotify.com)

Figure 13: Spotify Banner Advertisement

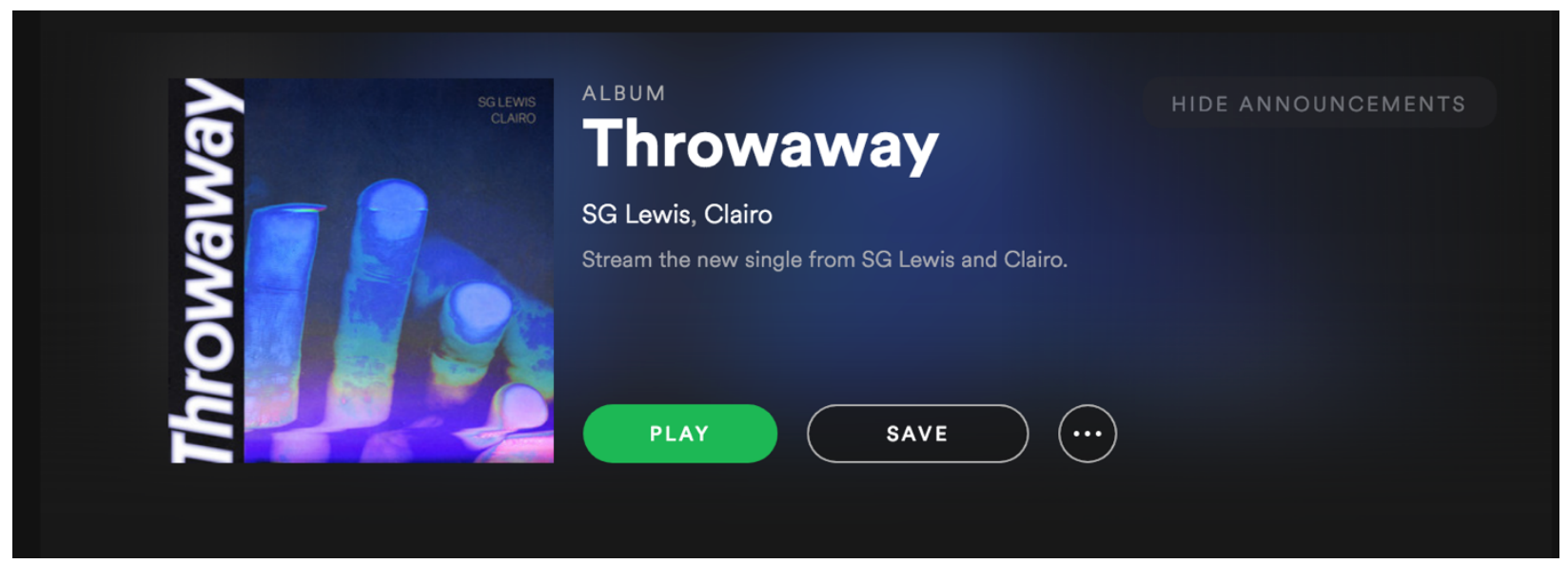

(Spotify.com) 
Figure 14: Spotify Video Advertisement-Jayden Smith x New Balance Brand

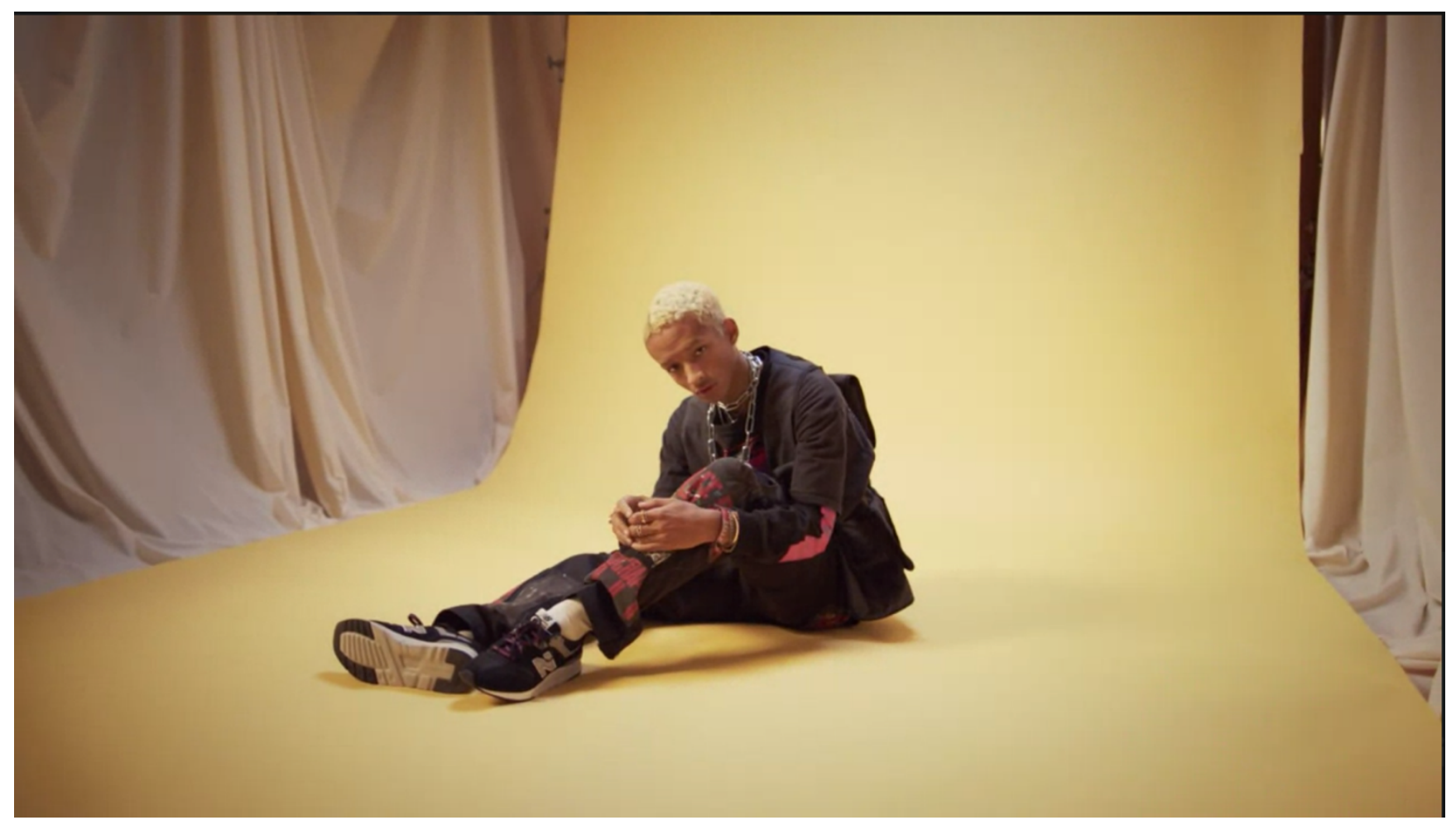

(Spotify.com)

Promotion is not exclusive to sponsored playlists, promotional messages or banner advertisements, but often takes form as album or cover art. Because SoundCloud artists have control of the content they distribute, they also have the power to upload their own cover art. The images in Figure 15 show the album cover art for the top songs listed in each of the different genre playlist. Some cover art features easily recognizable artists, like Ariana Grande, which are likely professionally created and distributed. However, a majority of the cover art for independent artists is hand-made or digitally made by the artists themselves. These illustrations allow for music listeners to get a better sense of who that artist is, whether it depicts an artist's physical appearance, personal style, or message they are trying to portray. A seemingly arbitrary image can have a great affect on the way that artist is perceived by the rest of the world. 
Figure 15: SoundCloud Cover Art

\section{Charts: Top 50}

The most played tracks on SoundCloud this week

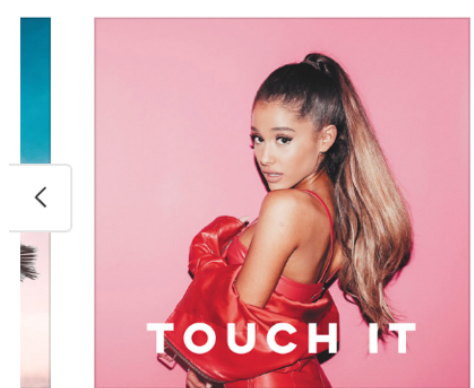

Disco

Top 50



Drum \& Bass Top 50

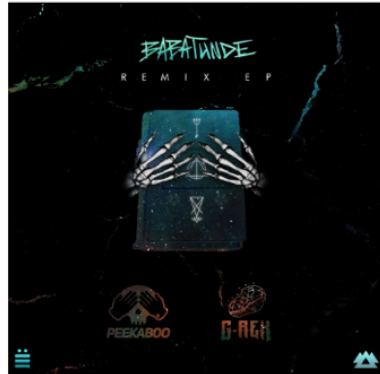

Dubstep Top 50

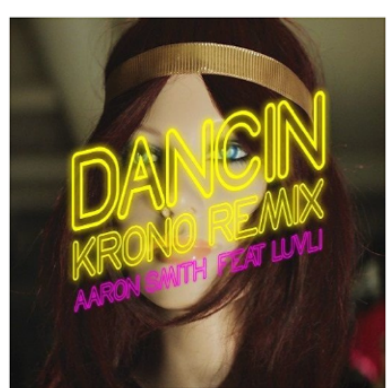

Electronic Top 50

\section{(Soundcloud.com)}

As shown in Figure 16, artists, Sam Smith \& Normani, not only top the playlist with their hit single "Dancing With a Stanger", but also serve as the cover art for Spotify's 'Today's Top Hits.' Famous R\&B singer, Jorja Smith, is singularly promoted on the cover of Spotify's 'Are \& Be' playlist and famous rap artist, Future, takes the cover of 'RapCaviar', with an added caption promoting his name along with popular rap artist, Logic. When these kinds of artists are featured, “their work is advertised prominently throughout the Spotify player in the 'discover' tab, on their main landing pages, or featured in 'exclusive' Spotify Sessions. These efforts represent an advanced promotional feedback loop that mixes user activity with interface design" (Morris \& Powers, 2015, p. 113). Whether the advertisements displayed are obvious targeted messages or discrete promotional features, these images have the power to influence the discovery of certain artists. 
MUSIC RECOMMENDATION ALGORITHMS

Figure 16: Spotify Cover Art

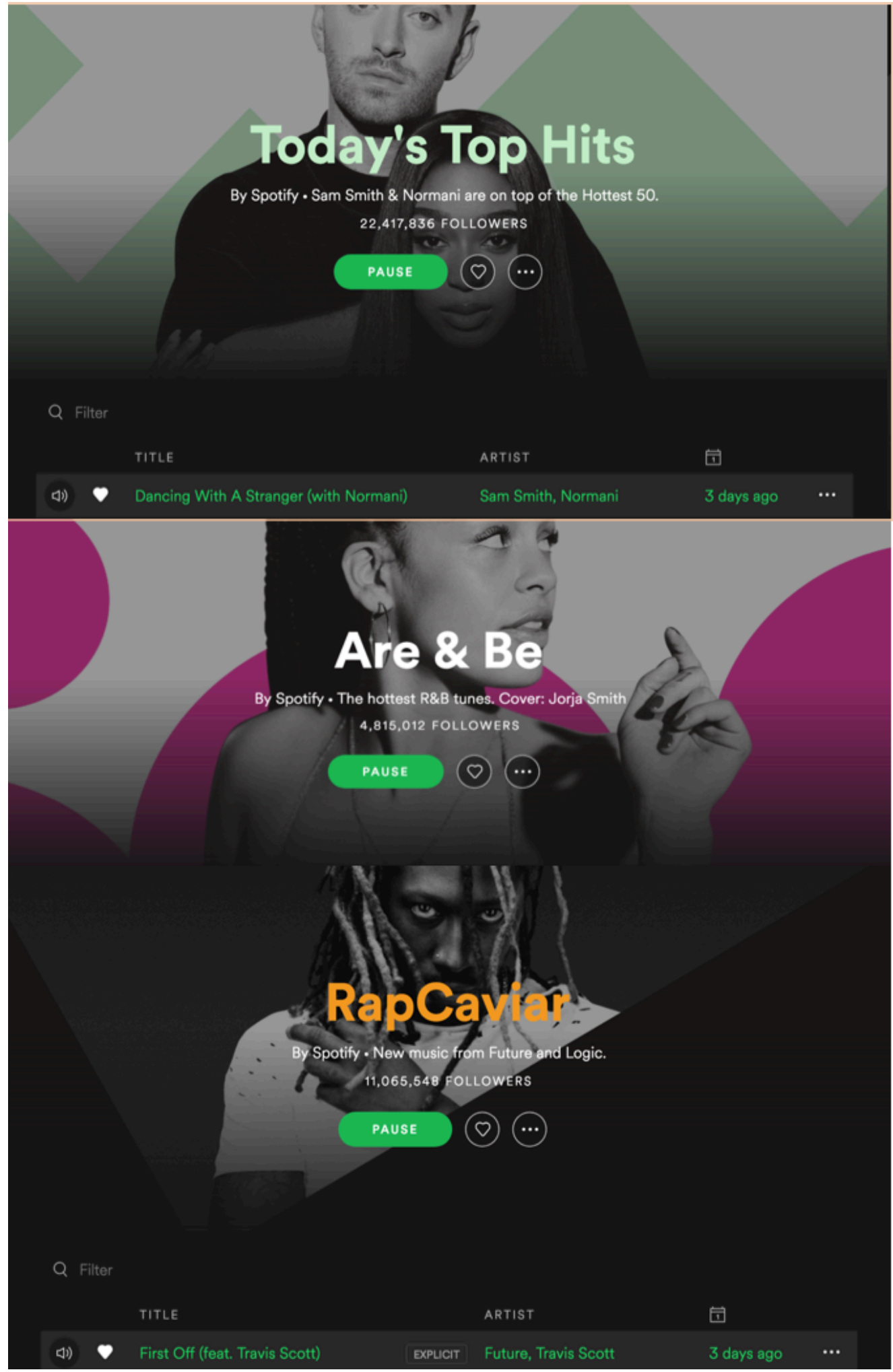

(Spotify.com) 


\section{MUSIC RECOMMENDATION ALGORITHMS}

If a new user immediately sees a banner advertisement promoting a new song or album on the home page of a streaming platform, it is likely for that user to be unsure at first about how the platform functions and naively listen to what is suggested. Not only in the case of new users, but when a sponsored playlist features an artist or band in its cover art and also throughout its song selections, it is inevitable for users to draw their attention to that artist. To better expose users to a wide variety of artists, it is not only important for there to be diversity throughout genre-based curated playlists, but also within advertisements.

\section{Discussion}

There are numerous limitations mentioned throughout the analysis of this experiment, including possibly subjective user registration information, generalization of genre categorization, mistagging of genres, and the influence of market trends within playlists; however, the most significant limitation is perhaps the overall limited quantity of listening data. As mentioned previously, unrepresentative recommendations could be the result of a "cold-start problem," or lack of listening history. Despite these drawbacks, this experiment has revealed a repetition of patterns across both platforms that cannot be dismissed. For one, both 'SoundCloud Weekly' and 'Discover Weekly' playlists reflected the current global sensation of Hip-hop/R\&B throughout a majority of recommendations rather than representing an equal representation of all genres listened to. Additionally, though the release year of genre-specific songs listened to were not necessarily sporadic amongst the two platforms, both recommendation algorithms generated recommendations that almost exclusively fell between the years 2018 and 2019. Lastly, the marketing of both SoundCloud and Spotify's Premium subscriptions and Spotify's commercial artists are likely to influence the behavior of users and have the power to persuade them to engage in the products being promoted. 


\section{MUSIC RECOMMENDATION ALGORITHMS}

While SoundCloud prides itself on supporting and cultivating independent artists and their creative freedom and Spotify boasts about the quality and accuracy of their recommendations, it is apparent that both recommendation algorithms intrinsically facilitate the perpetuation of a capitalist-driven marketplace, where popular, trendy, new music is favored despite the diversity of music listened to. This could be a consequence of the hierarchal structure of genres, subjective sequencing of songs, design of the interface and promotional materials, and many more matters of discernment. Because the makeup of recommendation algorithms remains invisible to the public, it is also feasible to suggest that these algorithms may not be exclusively powered by collaborative filtering, natural language processing, and convolution neural networks, but may also involve unknown components that are commercially favorable. In either case, music recommendation, at its core, requires a sense of contextualization in order to provide the most culturally attuned and individually accurate recommendations.

\section{Contextually-Aware Algorithms}

Although recommendation algorithms are high functioning in their ability to process massive amounts of data, they have not reached their full potential in satisfying users' personalized needs in terms of relevance and novelty. Constructing superior playlists for each user every single week is a challenging task, especially considering that this technology lacks a sense of psychological, cultural, and contextual awareness. "The algorithm that can judge the merits of new Gucci Mane, or intuit that you want to sing "A Thousand Miles" by Vanessa Carlton in the shower, has yet to be written" (Ugwu, 2016). Although recommendation algorithms may appear as if they can read our minds when a song is ironically recommended at the perceived "perfect moment", they are not quite as advanced as we'd like to think. 


\section{MUSIC RECOMMENDATION ALGORITHMS}

At this point in time, streaming services have assumed that a user is a fixed individual, "whose goals, needs, and tastes do not develop." (Pagano et al., 2016) Similar to the notion that every person has an appetite for different cuisine depending on their taste preference and contextual surrounding, every listener has an evolving "appetite" for specific kinds of music, creating a multi-dimensional taste profile for each listener. Spotify’s Product Manager of Personalized Programming, Ajay Kalia, believes that "it's important to recognize that a single music listener is usually many listeners" (Heath, 2015). This is why people will find that they share more interests with other people in the same or relatable situation than they do with past versions of themselves (Pagano, 2016). For instance, a college student at a party with friends will have more in common with another partying college student than they would with the person they were a few hours earlier, when studying for an exam or taking notes in class. Contextual recommendation ultimately allows for streaming service to nuance an understanding of each portion of a person's taste profile and in turn, regurgitate music through an awareness that is both flexible and adaptable. To improve contextual awareness, streaming platforms must collect and aggregate data points not just on everything that a listener consumes and interacts with, often referred to as extrinsic factors, but also a listener's personality and emotional state, known as intrinsic factors (Schedl et al., 2018).

Intrinsic factors derive from within and are naturally inherent in human nature. While an intrinsic factor like personality is likely to remain fairly consistent over time, an intrinsic factor like emotion is subject to change frequently in response to particular stimuli. "Musical content still contains various characteristics that affect the emotional state of the listener, such as lyrics, rhythm, and harmony, and the way how they affect the emotional state is highly subjective" (Schedl et al., 2018). Though personality and emotion have varying frequencies, 


\section{MUSIC RECOMMENDATION ALGORITHMS}

they are fundamental indicators of music taste. For example, extroverted personalities would likely have more in common with other extroverts rather than introverts. Those who are happy are likely to listen to songs with a fast tempo and in a major key, while those who are sad are likely to listen to songs with a slower tempo and in a minor key. These examples are merely basic defining characteristics that have the potential to be further developed into complex networks of emotional relation. In order to aggregate this intricate and very personal data, some sources have suggested that questionnaires could be administered to users, which would inquire about one's personality and personal taste (Schedl et al,. 2018). Others have proposed that streaming platforms collect and analyze data connected to users' social media profile (Schedl et al., 2018). Though both suggestions offer viable options, it is likely that questionnaires would be lengthy and inconsistent in response rate and that social media data would not only produce irrelevant recommendations, but also raise privacy concerns among users. Due to the fact that many people's music preference is contingent on their current psychological and cognitive states, many streaming platforms have aimed to not only categorize pieces of music with a genre tag, but also with an emotional tag. SoundCloud, for instance, displays the tag of a song in the top right corner of each song, using a hashtag followed by a defining characteristic (usually genre) of that song. With this said, even if streaming services are able to accurately predict the perceived emotion of a given song, the comprehension of a user's emotional state is vital to the matching of a user to song recommendation.

\section{Cultural Awareness}

Another aspect essential to the foundation of contextual awareness is cultural awareness. "While most humans share an inclination to listen to music, independent of their location or cultural background, the way music is performed, perceived, and interpreted evolves in a 


\section{MUSIC RECOMMENDATION ALGORITHMS}

culture-specific manner" (Schedl et al., 2018). The most obvious way that streaming platforms assign cultural elements to music are through playlists. For example, assigning the title "Reggae" to a selection of music that descends from Jamaica in the late 1960s, or "K-Pop" to a group of songs that characterize a young South Korean subculture create symbolic representations of specific cultures and shape the identities of certain populations. In a research project called CompMusic, funded by the European Research Council and coordinated by students at the Universitat Pompeu Fabra in Barcelona (Computational models, n.d.), researchers "identified geographical and cultural proximities that are reflected in music features, looking at outliers and misclassifications in a classification experiment using country as a target class" (Schedl et al. 2018). They ultimately found that "Vietnamese music was often confused with Chinese and Japanese" and "South African with Botswanese" (Schedl et al., 2018). Other than geographic location, culture can be defined on multiple levels, including language, historical background, native traditions, religion, nationality, race, gender, and age. Not only should the accuracy of cultural representations be prioritized by streaming platforms, but it is just as important for these platforms to create a diverse selection of genres, that reach far beyond the borders of a specific continent or country.

\section{Situational Awareness}

Possibly the most obvious requisite of contextually aware recommendation is situational awareness. Situation-aware music recommendation can include aggregated data that can specify location, time of day, day of week, weather, social context, user activity, mood, and much more. These defining characteristics are vital indications of a person's music preference in that moment and time. For instance, a music listener working out at the gym on a bright and sunny Monday morning would likely be drawn to more upbeat, optimistic music, while a music 


\section{MUSIC RECOMMENDATION ALGORITHMS}

listener working the last hour of their shift at a boring desk job on a rainy Friday afternoon would likely be drawn to slower, low-spirited music. Contextual awareness is not only sensitive to time or day or week, but also time that a song is released. One suggestion researchers offer is to time stamp ratings of songs, which separate the songs that may have been trendy years ago but are considered "out of style" in the present day. This implementation may however widen the gap between newly released songs and older songs, discouraging listeners from discovering songs from previous generations. In this case, time doesn't necessarily equate low ratings as older music may be relevant to certain audiences. As mentioned previously, music identity is not singular in nature and constantly evolves to specific contexts of life, whether that be time, location, or state of mind. While collaborative filtering, natural language processing, convolution neural networks, and outlier detection are fundamental in the construction of recommendation algorithms, contextual sensitivity has been a large missing piece in bringing algorithmic recommendation to the next level of accuracy. The technology that can perform these complex and sophisticated functions must be integrated into music streaming algorithms in order to incorporate this perceptive cognizance into recommendations; however, with this implementation, privacy concerns will inevitably arise amongst users.

\section{The Future of Music Recommendation}

The advancement of technological convergence, specifically the smartphone, has allowed for the collection of data points like location, motion, time of day, weather, nearby contacts, online activity, and much more. Not only does the smartphone have contextual capabilities, but "every car is about to ship with a SIM card, and that makes it a rolling hotspot" (Croll, 2015). The future of music listening will be tracked from our morning commutes, to the office, to the gym, to the kitchen, to the moment we lay our heads down at night. "Sensors, wearables, and 


\section{MUSIC RECOMMENDATION ALGORITHMS}

smarter playback systems can help, but some of these may invade privacy" (Croll, 2015). While the ability to track every aspect of a person's behavior and activity has been controversial in terms of intrusion, these advancements may be the next best thing for music recommendation. In fact, a team of Greek scientists, led by Dimitrios Adamos, at Aristotle University have pioneered the use of electroencephalogram (EEG) scanners for the purpose of music recommendation (Krywko, 2016) which are able to analyze the brain's electrical activity and discern how much a person likes a particular song. When these scanners are attached to the scalp, they can measure the intensity of dopamine a person releases while listening to music. In scientific terms, the auditory cortical regions of the brain, where sound is processed, and the frontal cortices, where the memory is stored, communicate to separate sounds into patterns. As people continue to listen to music over time, their brains are able to process these sound patterns and begin to predict what they will hear throughout a song. "Those predictions, when fulfilled, are fed into subcortical reward systems responsible for releasing dopamine. Fulfilled predication leads to dopamine release and thus pleasure" (Krywko, 2016). Adamos and his team used this technology to develop EEG wireless headsets that could analyze the interconnections between these brain regions and allow for individual music listeners to track their emotional experience while listening to music.

While EEG headsets currently exist in the market, this technology is still being tested and refined through prototyping. Most of the available headsets have not been practically confined to the size of an earbud and also cost upwards of $\$ 25,000$ per set. Once this technology is more practically designed and made cost-effectively, there is not much stopping mass-market services like Apple or Beats by Dre from implementing EEG technology into their headphones. There is also great potential for these companies to partner with streaming platforms and use this 


\section{MUSIC RECOMMENDATION ALGORITHMS}

technology to fuel the process of music listening and discovery. With the power to understand a user's emotional state at any given time, streaming platforms have the potential to transform the way music listening data is collected, analyzed, and curated into recommendations. This technology will completely terminate the use of skips, likes, dislikes, hearts, or stars as a form of communication between user and algorithm. Rather, these services would be provided automatic access to each user's electrical brain activity and process the patterns recorded using their recommendation algorithms. The intertwining of contextual filtering and collaborative filtering would likely produce the highest quality and precision of recommendations than ever before. If listeners don’t already feel that streaming services are reading their minds, they definitely will with this future technology.

\section{Conclusion}

It seems inevitable that music streaming platforms will continue to evolve as technology advances, especially in terms of personalized recommendation. Music listening is a complicated and ever-changing activity that humans invest a lot of time, emotion, and money into. Music is utilized as a means of universal expression, connection to others, and identity of oneself. The way music is recommended and discovered is a vital part of the music listening experience, nevertheless, there has yet to be a streaming platform that can provide recommendations that are accurate and satisfactory $100 \%$ of the time. While future technology does have the potential to improve the relevance and novelty of recommendations, it is important to account for the potential repercussions such evasive technology could have, let alone its potential lack of effectiveness and accessibility to society. I would suggest that the potential advantages and challenges of this technology are further studied and tested through future research. 


\section{MUSIC RECOMMENDATION ALGORITHMS}

At this point in time, this thesis aims to address and focus on the current challenges facing recommendation algorithms. Recommendation systems not only participate in the practices of music discovery, but ultimately shape the way users consume music. While providing a system that can filter and personalize over 30 million songs for each individual user, recommendation algorithms also privilege certain music, giving some artists and genres a better chance of being discovered than others. As gatekeepers of artists and music, recommendation algorithms must operate under the influence of cultural, social, and creative diversity, rather than the influence of capitalist interests that tend to cycle and promote the same "popular" sounds across genre and discovery playlists.

As articulated in the preceding experiment, the variation and accurate representation of genre, release year, artist status, and promotional content are fundamental to providing recommendations that meet the needs of users looking to discover relevant and novel content. The hierarchical sequencing of genres and songs, mistagging of genres, processing of user actions (skips, likes, dislikes, passive listening, etc.) as confidence measures, and irrelevant recommendation through drifting have all contributed to the inaccuracy and subjectivity projected to users by recommendation algorithms. As exhibited and supported throughout this thesis, much of the consequential subjectivity stems from human curation. The decisions made by expert curators may be driven by "gut," but cannot be separated from personal taste. The way human curators organize and label certain content (Western-based, English-language music) over other content (culturally diverse music), is processed into recommendation algorithms and ultimately affects the way users value certain music.

With the recent global shift in cultural trends, human curators have the potential to recalibrate these influential recommendation algorithms. Through logic, reasoning, and 


\section{MUSIC RECOMMENDATION ALGORITHMS}

sensitivity, qualities only characteristic of humans, recommendations can continually expand in genre, style, language, and nationality. It seems imperative that human curators work hand in hand with algorithms, which often inform curators of both larger and also subtler trends.

However, it is also apparent that the use of complex user data can be used to drive promotional motivations, like fragmenting listeners based on perceived identities, which causes the segregation of users into superficial "filter bubbles." Although not perfected, algorithms have great potential to create lasting connections between artists and fans, further diversify the curation of content within playlists, and encourage users to discover the music and artists they never knew they would love. Spotify's Nordic Head of Shows and Editorial, Daniel Breitholtz, says that 'Discover Weekly' recommendations “are based on your listening behaviours, and should mirror your music taste. If they are not working for you it's not our fault! It's you!" (Dredge, 2017). Daniel Breitholtz must be misinformed. 
MUSIC RECOMMENDATION ALGORITHMS

References

About RIAA. (n.d.). Retrieved December 04, 2018, from https://www.riaa.com/about-riaa/

Ahlers, M., Grünewald-Schukalla, L., Lücke, M., \& Rauch, M. (2018). Big Data und Musik Jahrbuch für Musikwirtschafts- und Musikkulturforschung 1/2018. Wiesbaden: Springer Fachmedien Wiesbaden GmbH.

Amoore, L. (2011). Data Derivatives. Theory, Culture \& Society,28(6), 24-43. doi: $10.1177 / 0263276411417430$

Battan, C. (2019, January 31). The Messy Story of How SoundCloud Rap Took Over Everything. Retrieved from https://www.gq.com/story/soundcloud-rap-boom-times

Beaumont-Thomas, B., \& Snapes, L. (2018, October 05). Has 10 years of Spotify ruined music? Retrieved from https://www.theguardian.com/music/2018/oct/05/10-years-of-spotify$\underline{\text { should-we-celebrate-or-despair }}$

Caulfield, K. (2018, January 03). U.S. Music Consumption Up 12.5\% in 2017, R\&B/Hip-Hop Is Year's Most Popular Genre. Retrieved from https://www.billboard.com/articles/columns/chart-beat/8085975/us-music-consumptionup-2017-rb-hip-hop-most-popular-genre 


\section{MUSIC RECOMMENDATION ALGORITHMS}

Celma, O. (2010) Music Recommendation and Discovery: The Long Tail, Long Fail, and Long Play in the Digital Music Space. Berlin: Springer.

Celma, O. \& Lamere, P. (2011). If you like Radiohead, you might like this article. AI Magazine. 32(3), 57-66. http://helios.uta.fi/docview/908412528?accountid=14242.

Ciocca, S. (2018, June 21). How Does Spotify Know You So Well? - Member Feature Stories Medium. Retrieved from https://medium.com/s/story/spotifys-discover-weekly-how$\underline{\text { machine-learning-finds-your-new-music-19a41ab76efe }}$

Computational models for the discovery of the World's Music. (n.d.). Retrieved from https://compmusic.upf.edu/

Croll, A. (2015, September 15). Music science. Retrieved from https://www.oreilly.com/ideas/music-science

Davis, G. (2014, July 21). Future of Music Coalition. Retrieved from https://futureofmusic.org/blog/2014/07/21/understanding-soundclouds-copyright-woes

Deahl, D. (2019, February 19). SoundCloud now helps artists self-distribute music to Spotify and other streaming platforms. Retrieved from https://www.theverge.com/2019/2/19/18231602/soundcloud-spotify-self-distribution$\underline{\text { streaming-platforms-music }}$ 


\section{MUSIC RECOMMENDATION ALGORITHMS}

Deahl, D. (2018, September 20). Spotify will now let artists directly upload their music to the platform. Retrieved from https://www.theverge.com/2018/9/20/17879840/spotify-artistdirect-upload-independent-music

Discover Hits From Around the World With Spotify's Global Cultures Initiative. (2018, November 28). Retrieved from https://newsroom.spotify.com/2018-09-28/discover-hitsfrom-around-the-world-with-spotifys-global-cultures-initiative/

Dredge, S. (2018, March 29). News. Retrieved from https://musically.com/2018/03/29/pandora$\underline{\text { starts-testing-personalised-playlists/ }}$

Dredge, S. (2017, November 29). Analysis. Retrieved from https://musically.com/2017/11/29/spotify-playlists-skip-rates-nf/

Eder, S. (2018, June 28). How Can We Eliminate Bias In Our Algorithms? Retrieved from https://www.forbes.com/sites/theyec/2018/06/27/how-can-we-eliminate-bias-in-ouralgorithms/\#3f0f0e91337e

Eriksson, M., \& Johansson, A. (2017). Tracking Gendered Streams. Culture Unbound: Journal of Current Cultural Research,9(2), 163-183. doi:10.3384/cu.2000.1525.1792163 


\section{MUSIC RECOMMENDATION ALGORITHMS}

Flynn, M. (2016). Accounting for Listening: How music streaming has changed what it means to listen. Musical and Media Connectivities: Practices, Circulation, Interactions, 6.

Galvanize. (2016, August 22). Ever Wonder How Spotify Discover Weekly Works? Data Science. Retrieved from https://blog.galvanize.com/spotify-discover-weekly-datascience/

Global streaming music subscribers 2017 | Statistic. (2017, February). Retrieved from https://www.statista.com/statistics/669113/number-music-streaming-subscribers/

Hall, P. (2018, November 05). Spotify is the best streaming service, but its competitors aren't far behind. Retrieved from https://www.digitaltrends.com/music/best-music-streaming$\underline{\text { services/ }}$

Heath, A. (2015) Spotify has a secret 'taste profile' on everyone, and they showed me mine. Retrieved from http://www.businessinsider.com/how-spotify-taste-profiles-work-2015$\underline{\text { 9?international }=\text { trueandr }=\mathrm{USand} \mathrm{IR}=\mathrm{T}}$

Horn, L. (2014, June 16). How SoundCloud Changed Music Forever. Retrieved from https://gizmodo.com/how-soundcloud-changed-music-forever-1588811594

Hu, C. (2018, September 11). Paid Music Streaming Subscribers Surpass 50 Million in US, But There's a Twist: Exclusive. Retrieved from 


\section{MUSIC RECOMMENDATION ALGORITHMS}

https://www.billboard.com/articles/business/8474560/paid-music-streaming-subscriberssurpass-50-million-us-exclusive

Hussein, W. (2018, August 09). Apple Music is reportedly making a playlist full of songs your friends listen to. Retrieved from https://www.thefader.com/2018/08/09/apple-music$\underline{\text { friends-mix-personalized-playlists }}$

Ingham, T. (2016, February 08). How much does SoundCloud really owe the music business? Retrieved from https://www.musicbusinessworldwide.com/how-much-does-soundcloudowe-the-music-business/

James, A. (2017, January 13). A Journey Down the Rabbit Hole of SoundCloud Related Tracks. Retrieved from https://djbooth.net/features/2017-01-13-soundcloud-related-tracks

Kastrenakes, J. (2019, March 26). Spotify is personalizing more playlists to individual users. Retrieved from https://www.theverge.com/2019/3/26/18282549/spotify-personalizedplaylists-curation-more-songs

Kelly, K. (2016, February 14). A Brief History of American Payola. Retrieved from https://noisey.vice.com/en us/article/64y8y9/a-brief-history-of-american-payola

Knibbe, J. (2017, March 07). Is Music Personal? - Deezer I/O. Retrieved from https://deezer.io/is-music-personal-315106ec0179 


\section{MUSIC RECOMMENDATION ALGORITHMS}

Kachkach, A. (2016). Analyzing user behavior and sentiment in music streaming services. $K T H$ ROYAL INSTITUTE OF TECHNOLOGY SCHOOL OF COMPUTER SCIENCE AND COMMUNICATION.

Kostanje, E. (2018, September 10). SoundCloud Takes on Spotify by Adding Weekly Personalized Playlist. Retrieved from https://www.pastemagazine.com/articles/2018/09/soundcloud-takes-on-spotify-adds$\underline{\text { weekly-personaliz.html }}$

Kuoppa, A. (2018). Recommended by Algorithm: Relevance, Affordances and Agency of Music Recommender Systems(Unpublished master's thesis). University of Tampere.

Krywko, J. (2016, October 18). The only way an algorithm can tell if you really like a song is by scanning your brain. Retrieved from https://qz.com/812498/eeg-brain-scans-can-tell-ifyou-like-a-song-better-than-any-music-streaming-service-algorithm/

Lamere, P. (2014, May 06). The Skip. Retrieved from https://musicmachinery.com/2014/05/02/the-skip/

Light, B., Burgess, J., \& Duguay, S. (2016). The walkthrough method: An approach to the study of apps. New Media \& Society,20(3), 881-900. doi:10.1177/1461444816675438 


\section{MUSIC RECOMMENDATION ALGORITHMS}

Lops, P., Gemmis, M. D., \& Semeraro, G. (2010). Content-based Recommender Systems: State of the Art and Trends. Recommender Systems Handbook,73-105. doi:10.1007/978-0-38785820-3_3

Lynch, J. (2018, January 04). For the first time in history, hip-hop has surpassed rock to become the most popular music genre, according to Nielsen. Retrieved from https://www.businessinsider.com/hip-hop-passes-rock-most-popular-music-genre$\underline{\text { nielsen-2018-1 }}$

Marks, C. (2017, September 13). How a Hit Rap Song Happens Now. Retrieved from https://www.vulture.com/2017/09/spotify-rapcaviar-most-influential-playlist-in$\underline{\text { music.html }}$

Max. (2014, December 7). 7 Ways to Promote Your SoundCloud Music. Retrieved from https://soundcloudreviews.org/7-ways-promote-soundcloud-music/

McClain, J., \& McClain, A. (2012). Retrieved from https://philadelphiaencyclopedia.org/archive/american-bandstand/

McIntyre, H. (2018, March 21). The Piracy Sites That Nearly Destroyed The Music Industry: What Happened To Napster. Retrieved from https://www.forbes.com/sites/hughmcintyre/2018/03/21/what-happened-to-the-piracysites-that-nearly-destroyed-the-music-industry-part-1-napster/\#f6eeec12293b 


\section{MUSIC RECOMMENDATION ALGORITHMS}

McIntyre, H. (2018, March 21). The Piracy Sites That Nearly Destroyed The Music Industry: What Happened To Kazaa. Retrieved from https://www.forbes.com/sites/hughmcintyre/2018/03/21/xxx-what-happened-to-thepiracy-sites-that-nearly-destroyed-the-music-industry-part-3-kazaa/\#42d92e5655fa

McIntyre, H. (2017, December 11). How Longer Albums And Streaming Giants Are Manipulating The Charts. Retrieved from https://www.forbes.com/sites/hughmcintyre/2017/08/17/how-longer-albums-and$\underline{\text { streaming-giants-are-manipulating-the-charts/ }}$

Meier, L. M., \& Manzerolle, V. R. (2018). Rising tides? Data capture, platform accumulation, and new monopolies in the digital music economy. New Media \& Society,21(3), 543-561. doi: $10.1177 / 1461444818800998$

Morris, J. W., \& Powers, D. (2015). Control, curation and musical experience in streaming music services. Creative Industries Journal,8(2), 106-122.

doi:10.1080/17510694.2015.1090222

Newton, C. (2019, January 25). YouTube says it will recommend fewer videos about conspiracy theories. Retrieved from https://www.theverge.com/2019/1/25/18197301/youtubealgorithm-conspiracy-theories-misinformation 


\section{MUSIC RECOMMENDATION ALGORITHMS}

Novet, J. (2014, August 06). Spotify intern dreams up better music recommendations through deep learning. Retrieved from https://venturebeat.com/2014/08/06/spotify-intern-dreamsup-better-music-recommendations-through-deep-learning/

Owsinski, B. (n.d.). New Analysis Calculates, Compares Per Stream Payouts From Spotify, Apple Music, Pandora, YouTube and More. Retrieved from https://www.hypebot.com/hypebot/2017/07/new-analysis-calculates-compares-per$\underline{\text { stream-payouts-from-spotify-apple-music-pandora-youtube-and-mor.html }}$

Pagano, R., Cremonesi, P., Larson, M., Hidasi, B., Tikk, D., Karatzoglou, A., \& Quadrana, M. (2016). The Contextual Turn. Proceedings of the 10th ACM Conference on Recommender Systems - RecSys 16. doi:10.1145/2959100.2959136

Pasick, A. (2015, December 22). The magic that makes Spotify's Discover Weekly playlists so damn good. Retrieved from https://qz.com/571007/the-magic-that-makes-spotifysdiscover-weekly-playlists-so-damn-good/

Payne, O. (2018, September 20). Spotify Will Now Allow Independent Artists To Upload Music Directly To The Platform. Retrieved from https://www.forbes.com/sites/ogdenpayne/2018/09/20/spotify-will-now-allowindependent-artists-to-upload-music-directly-to-the-platform/\#6b6660966d45 


\section{MUSIC RECOMMENDATION ALGORITHMS}

Prey, R. (2018). Nothing personal: algorithmic individuation on music streaming platforms. Media, Culture \& Society, 40(7), 1086-

1100. https://doi.org/10.1177/0163443717745147

Promotion - FAQ - Spotify for Artists. (n.d.). Retrieved from https://artists.spotify.com/faq/promotion\#how-do-i-submit-music-to-your-editorial-team

Pybis, E. (2018, June 27). Hip Hop Raised Me. Retrieved from https://www.quora.com/Whendid-hip-hop-become-the-most-popular-genre-in-the-world

Rohan. (2018, August 13). Recommendation Systems: How Spotifiers Discover New Songs Weekly. Retrieved from https://acadgild.com/blog/recommendation-systems-spotify

Relevant. (n.d.). Retrieved from https://www.merriam-webster.com/dictionary/relevant

Ryan, P. (2018, January 04). Rap overtakes rock as the most popular genre among music fans. Here's why. Retrieved from https://www.usatoday.com/story/life/music/2018/01/03/rapovertakes-rock-most-popular-genre-among-music-fans-heres-why/990873001/

Sanchez, D. (2018, April 16). SoundCloud's Financial Losses Are Way, Way Worse Than We Thought. Retrieved from https://www.digitalmusicnews.com/2018/04/13/soundcloud2016-financials/ 


\section{MUSIC RECOMMENDATION ALGORITHMS}

Schedl, M., Zamani, H., Chen, C., Deldjoo, Y., \& Elahi, M. (2018). Current challenges and visions in music recommender systems research. International Journal of Multimedia Information Retrieval, 7(2), 95-116. doi:10.1007/s13735-018-0154-2

Seto, E. (2018, September 11). SoundCloud Weekly bids to displace Discover Weekly as a music discovery platform. Retrieved from http://www.fouroverfour.jukely.com/news/soundcloud-weekly-music-discovery/

Seydel, R. (2016, March 6). SoundCloud Promotion: 8 Ways to Actually Get Heard. Retrieved from https://blog.landr.com/8-ways-actually-get-heard-soundloud/

Singer, N. (2017, December 20). Listen to Pandora, and It Listens Back. Retrieved from https://www.nytimes.com/2014/01/05/technology/pandora-mines-users-data-to-bettertarget-ads.html

Singleton, M. (2017, November 14). Spotify's RapCaviar turned the playlist into a movement, now Viva Latino is next. Retrieved from https://www.theverge.com/2017/11/14/16635562/spotify-viva-latino-rap-caviar-playlist$\underline{\text { music-video-redesign }}$

Snickars, P. (2017). More of the Same - On Spotify Radio. Culture Unbound: Journal of Current Cultural Research, 9(2), 184-211. doi:10.3384/cu.2000.1525.1792184 


\section{MUSIC RECOMMENDATION ALGORITHMS}

SoundCloud Premier. (n.d.). Retrieved from https://blog.soundcloud.com/tag/soundcloudpremier/

Srnicek, N. (2017). Platform Capitalism. Cambridge: Polity Press.

Stats basics. (n.d.). Retrieved from https://help.soundcloud.com/hc/en-us/articles/115003568708$\underline{\text { Stats-basics }}$

The history of DJing. (2012). Retrieved from https://radiosolution.info/newsletter/website pages/history of DJ.html

The Media Show, BONUS Rob Stringer, Sony Music CEO. (2018, October 03). Retrieved from https://www.bbc.co.uk/programmes/p06mvj32

Timberg, S. (2016, June 10). Spotify is making you boring: When algorithms shape music taste, human curiosity loses. Retrieved from https://www.salon.com/2016/06/10/spotify is making you boring when algorithms sh ape music taste human_curiosity_loses/

Titlow, J. P. (2018, April 13). It's Official: The Beatles Coming To Spotify, Apple Music, And More. Retrieved from https://www.fastcompany.com/3054965/its-official-the-beatlesare-coming-to-spotify-apple-music-and-more 


\section{MUSIC RECOMMENDATION ALGORITHMS}

Ugwu, R. (2016, July 13). The Playlist Professionals At Apple, Spotify, And Google. Retrieved from https://www.buzzfeed.com/reggieugwu/the-unsung-heroes-of-the-music-streaming$\underline{\text { boom }}$

Washenko, A. (2018, September 10). SoundCloud Weekly is the audio service's pitch for personalized discovery. Retrieved from https://rainnews.com/soundcloud-weekly-is-theaudio-services-pitch-for-personalized-discovery/

Wikipedia contributors. (2018, October 23). Music magazine. In Wikipedia, The Free Encyclopedia. Retrieved 16:31, March 27, 2019, from https://en.wikipedia.org/w/index.php?title=Music magazine\&oldid=865428855

Wortham, J. (2017, August 01). If SoundCloud Disappears, What Happens to Its Music Culture? Retrieved from https://www.nytimes.com/2017/08/01/magazine/if-soundclouddisappears-what-happens-to-its-music-culture.html 